\title{
Squeezing a drop of nematic liquid crystal with strong elasticity effects
}

Cite as: Phys. Fluids 31, 083107 (2019); https://doi.org/10.1063/1.5110878

Submitted: 21 May 2019 . Accepted: 22 July 2019 . Published Online: 22 August 2019

J. R. L. Cousins (D), S. K. Wilson (D), N. J. Mottram (D), D. Wilkes, and L. Weegels
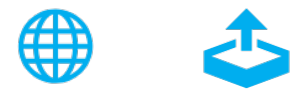

\section{ARTICLES YOU MAY BE INTERESTED IN}

The rising velocity of a slowly pulsating bubble in a shear-thinning fluid

Physics of Fluids 31, 083103 (2019); https://doi.org/10.1063/1.5108812

Particle and rigidized red blood cell concentration distributions in microchannel flows

Physics of Fluids 31, 082006 (2019); https://doi.org/10.1063/1.5111201

Direct numerical simulation of a three-dimensional spatially evolving compressible mixing layer laden with particles. II. Turbulence anisotropy and growth rate

Physics of Fluids 31, 083303 (2019); https://doi.org/10.1063/1.5110990

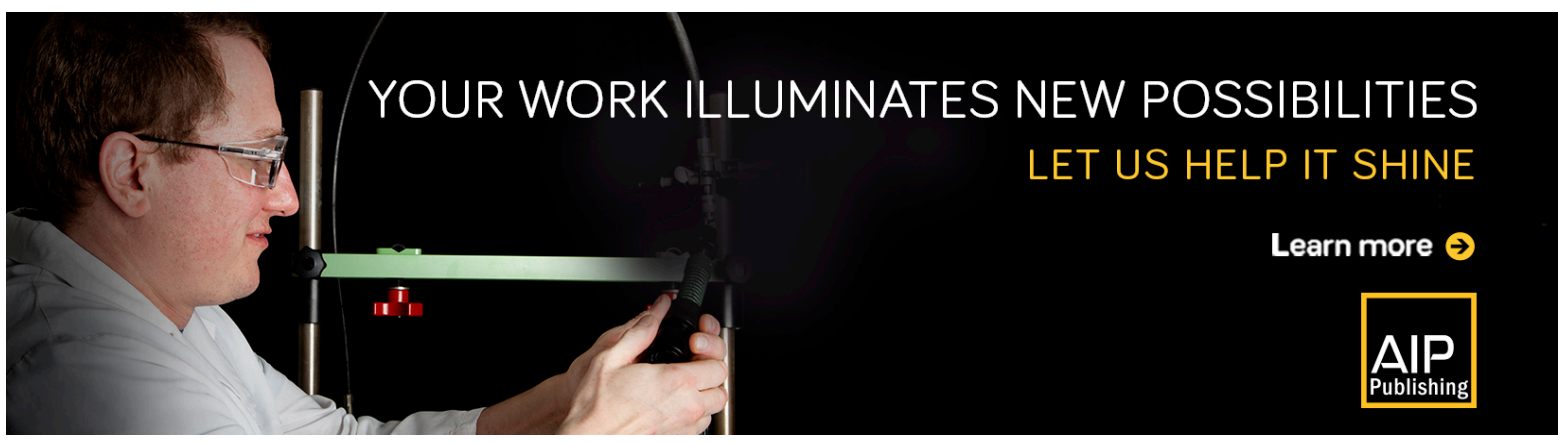




\title{
Squeezing a drop of nematic liquid crystal with strong elasticity effects
}

\author{
Cite as: Phys. Fluids 31, 083107 (2019); doi: 10.1063/1.5110878 \\ Submitted: 21 May 2019 - Accepted: 22 July 2019 • \\ Published Online: 22 August 2019
}

\section{J. R. L. Cousins, ${ }^{1, a)}$ (D) S. K. Wilson, ${ }^{1, b)}$ (D) N. J. Mottram, ${ }^{1, c)}$ (D) D. Wilkes, ${ }^{2, d)}$ and L. Weegels ${ }^{2, e)}$}

\author{
AFFILIATIONS \\ 'Department of Mathematics and Statistics, University of Strathclyde, Livingstone Tower, 26 Richmond Street, \\ Glasgow G1 1XH, United Kingdom \\ ${ }^{2}$ Merck KGaA, Frankfurter Str. 250, Darmstadt 64293, Germany \\ a) joseph.cousins@strath.ac.uk \\ b) Author to whom correspondence should be addressed: s.k.wilson@strath.ac.uk \\ c)nigel.mottram@strath.ac.uk \\ d)david.wilkes@merckgroup.com \\ e)leo.weegels@merckgroup.com
}

\begin{abstract}
The One Drop Filling (ODF) method is widely used for the industrial manufacture of liquid crystal devices. Motivated by the need for a better fundamental understanding of the reorientation of the molecules due to the flow of the liquid crystal during this manufacturing method, we formulate and analyze a squeeze-film model for the ODF method. Specifically, we consider a nematic squeeze film in the asymptotic regime in which the drop is thin, inertial effects are weak, and elasticity effects are strong for four specific anchoring cases at the top plate and the substrate (namely, planar, homeotropic, hybrid aligned nematic, and $\pi$-cell infinite anchoring conditions) and for two different scenarios for the motion of the top plate (namely, prescribed speed and prescribed force). Analytical expressions for the leading- and first-order director angles, radial velocity, vertical velocity, and pressure are obtained. Shear and couple stresses at the top plate and the substrate are calculated and are interpreted in terms of the effect that flow may have on the alignment of the molecules at the plates, potentially leading to the formation of spurious optical defects ("mura").
\end{abstract}

Published under license by AIP Publishing. https://doi.org/10.1063/1.5110878

\section{INTRODUCTION}

The industrial manufacture of liquid crystal devices involves a number of different processes, a key one of which involves filling a liquid crystal between two solid parallel plates of glass or plastic. There are two basic methods for doing this, namely, capillary filling and One Drop Filling (ODF). In the capillary filling method, the two parallel plates are first fixed together so that there is a prescribed gap between them. The liquid crystal is then introduced into this gap by capillary forces, usually under a vacuum, until the device is filled. ${ }^{1-3}$ For standard liquid crystal materials and device gaps (typically less than $10 \mu \mathrm{m}$ ), the relatively long time scale of capillary filling (typically $1-2$ days ${ }^{2,4}$ ) means that using this method can be a key factor limiting the rate of device production. In the ODF method, an array of drops of the liquid crystal are placed on one of the plates, here called the substrate, and the second plate, here called the top plate, is lowered into position, squeezing the drops until they coalesce to form a continuous film which fills the device. ${ }^{3,5}$ The motion of the top plate is stopped when the required gap between the plates is attained. The relatively short time scale of the ODF method (originally typically 1-2 hours, ${ }^{2,4}$ but now much shorter than this) means that devices can be produced at a much faster rate using this method rather than the capillary filling method. As well as moving from the capillary filling method to the ODF method, manufacturers seek to further reduce manufacturing time by increasing the speed of the top plate, which increases flow speeds, but this is often done without a clear understanding of the possible consequences of flow-driven reorientation and subsequent misalignment of the molecules at the plates. Indeed, spurious optical effects, termed "mura" (a Japanese word meaning irregularity or lack of uniformity), have been reported, which degrade liquid crystal device performance. ${ }^{6-8}$ The misalignment of the molecules at the 
plates due to the flow of the liquid crystal is one possible mechanism for the appearance of mura. ${ }^{8}$ We have recently proposed reducing the occurrence of mura by using smaller drops and by moving the top plate more slowly. ${ }^{8}$ The effect of these changes is to reduce the flow speed during squeezing, which in turn reduces the degree of molecular reorientation within the device; however, the time taken to manufacture the device increases. In this work, we will consider a mathematical model for the squeezing and spreading of a drop of liquid crystal between two parallel plates which occurs during the ODF method. Specifically, we will consider smaller drops and slower plate speeds than those typical of the current ODF method in order to provide insight into the possible mechanisms of molecular misalignment in this possible future manufacturing regime. In particular, an understanding of the behavior of the liquid crystal in this regime could potentially lead to improvements in manufacturing efficiency.

In this work, we will consider nematic liquid crystalline materials, which are hereafter termed nematics, since it is these materials that are used in the ODF method of liquid crystal device manufacture. In nematics, there is long-range order of the molecular orientation but no positional order of the molecules. There are many approaches to modeling nematics, ranging from atomistic and molecular models ${ }^{9-11}$ to continuum models. ${ }^{12,13}$ For the system we consider, for which variations occur on the micron length scale and the millisecond time scale, it is appropriate to use a continuum model. The standard continuum model for nematics uses the average molecular direction as a dependent variable, which is mathematically described by a unit vector $\mathbf{n}$ and is known as the director. ${ }^{12,14}$ Describing the dynamics of a nematic involves modeling the coupling of the director $\mathbf{n}$ and fluid velocity $\mathbf{u}$, which is mathematically captured by the Ericksen-Leslie equations ${ }^{15,16}$ subject to appropriate boundary conditions. The Ericksen-Leslie equations represent statements of the conservation of mass, linear momentum, and angular momentum and have successfully been used to model many different fundamental effects and industrially relevant processes in liquid crystals. ${ }^{12}$

The flow of the nematic during the ODF method after the top plate has made contact with the drops is similar to the classical squeeze-film problem in Newtonian fluid dynamics. ${ }^{17}$ In this work, we therefore consider a nematic squeeze-film problem in which director orientation and flow are coupled, using the Ericksen-Leslie equations in place of the Navier-Stokes equations used to describe the Newtonian problem. Specifically, we consider a layer of nematic confined between two parallel plates and squeezed by the motion of the top plate.

In Sec. II, we formulate a squeeze-film model for the ODF method in which we assume an idealized form of the shape of the nematic drop, for four specific director anchoring cases at the substrate and the top plate and for two different scenarios for the motion of the top plate. After nondimensionalizing the governing equations and boundary conditions, we consider the asymptotic regime in which elasticity effects are much stronger than the viscous ones and solve the resulting system of equations and boundary conditions in order to better understand the effects of director-flow coupling. In particular, we obtain analytical expressions for the director, velocity, and pressure, as well as the force on the top plate (when the speed of the top plate is prescribed) and the speed of the top plate (when the force on the top plate is prescribed).

\section{MODEL FORMULATION}

In order to model the squeezing and spreading of a nematic drop which occurs during the ODF method, we consider a geometrically simplified problem. We assume that at some time $t<0$ the top plate and the drop make contact and that by time $t=0$ any transient initial effects arising from starting the squeezing process can be ignored. For $t \geq 0$, we assume that the drop of nematic is cylindrical in shape, lies between the moving top plate at $z=h(t)$ and the fixed substrate at $z=0$, and has radius $R(t)$ and height equal to the height of the top plate $h(t)$, as shown in Fig. 1. The top plate and substrate are assumed to have a fixed area, denoted by $A$, where we assume that throughout squeezing $A>\pi R(t)^{2}$. The constant volume of the drop of nematic $V$ is given in terms of its radius and height by

$$
V=\pi R(t)^{2} h(t) .
$$

By conservation of mass, the outer boundary of the nematic, $r=R(t)$, moves outward radially as the top plate moves toward the substrate. We consider two different ambient pressures: the internal ambient pressure between the top plate and the substrate, denoted by $p_{\mathrm{I}}$, and the external ambient pressure above the top plate, denoted by $p_{\mathrm{E}}$. Typically, manufacturing processes are carried out in a vacuum to avoid the formation of air bubbles, ${ }^{2}$ so in what follows we often set $p_{\mathrm{I}}=p_{\mathrm{E}}=0$.

To model the squeezing and spreading of the nematic drop, we use the Ericksen-Leslie equations ${ }^{12,14-16}$ to describe the dynamics of the director orientation, velocity, and pressure. At both the substrate and top plate, we impose the standard no-slip and no-penetration conditions for the velocity. In addition, we assume that the director is at a fixed angle to the surface normal, termed an infinite anchoring condition. ${ }^{12}$ Although the analysis presented below is valid for any fixed angles of the director at the substrate and top plate, we will focus on four specific cases of infinite anchoring that are commonly used in nematic devices, namely, planar, homeotropic, hybrid aligned nematic (HAN), and $\pi$-cell anchoring. ${ }^{18}$ All of these anchoring cases involve various combinations of the director being aligned parallel or perpendicular to the boundaries. A boundary condition in

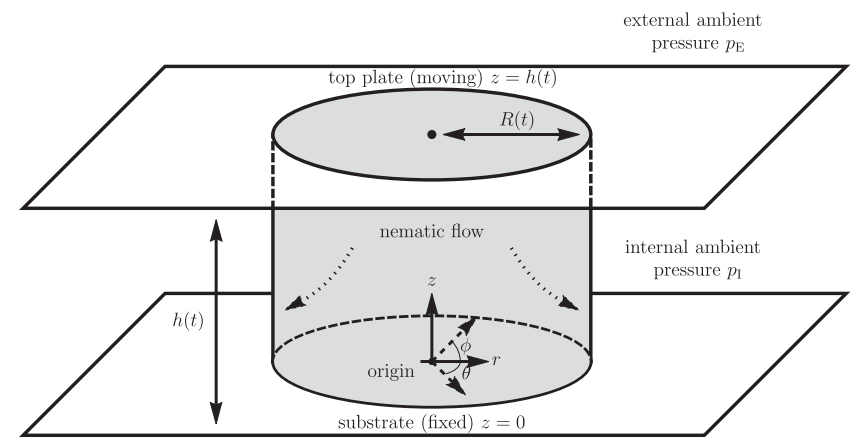

FIG. 1. A schematic diagram of a nematic squeeze-film problem consisting of a cylindrical drop of radius $R(t)$ of nematic (gray) between a moving top plate at $z=h(t)$ and a fixed substrate at $z=0$. The motion of the top plate induces a flow of the nematic as indicated. The internal ambient pressure $p_{l}$, external ambient pressure $p_{\mathrm{E}}$, the director angle $\phi$, and the axisymmetric coordinates used to describe the problem are also indicated. 
which the director is perpendicular to the boundary corresponds to a situation in which a mechanical or chemical treatment of the boundary (for example, the addition of a surfactant such as lecithin ${ }^{18}$ ) forces the nematic molecules at the boundary to orient parallel to the surface normal. On the other hand, a boundary condition in which the director is parallel to the boundary corresponds to a situation in which the boundary is coated with a polymer (for example, PVA or polyimide ${ }^{18}$ ) which forces the nematic molecules at the boundary to orient perpendicular to the surface normal (i.e., to lie in the plane of the boundary). In the absence of flow, the orientation of the director at the boundary is planar degenerate, but in the presence of flow, the director will align with the direction of flow. In particular, for the radial flow considered in this work, this will align the director in the radial direction.

In addition to the four anchoring cases, two different scenarios for the motion of the top plate will be studied. The first scenario corresponds to the current ODF method in which the top plate is moved downwards at a prescribed constant speed, and is hereafter referred to as the "prescribed speed" scenario. The second scenario corresponds to the situation in which the top plate moves downwards under a prescribed force (for example, its own weight) and is hereafter referred to as the "prescribed force" scenario.

\section{A. The Ericksen-Leslie equations}

We assume that both the director field and the velocity remain axisymmetric so that all dependent variables are independent of the azimuthal angle, $\theta$, shown in Fig. 1 . The director is assumed to lie in the $r-z$-plane and can therefore be described by an angle $\phi$ between the director and the radial direction, also shown in Fig. 1. We therefore write the director, pressure, and velocity in the form

$$
\begin{aligned}
& \mathbf{n}=\cos (\phi(r, z, t)) \hat{\mathbf{e}}_{r}+\sin (\phi(r, z, t)) \hat{\mathbf{e}}_{z}, \\
& \mathbf{u}=u(r, z, t) \hat{\mathbf{e}}_{r}+w(r, z, t) \hat{\mathbf{e}}_{z}, \\
& p=p(r, z, t),
\end{aligned}
$$

where $u$ is the component of velocity in the radial direction $\hat{\mathbf{e}}_{r}$ and $w$ is the component of velocity in the vertical direction $\hat{\mathbf{e}}_{z}$. The Ericksen-Leslie equations for the director, velocity, and pressure in the form of Eqs. (2)-(4), respectively, are

$$
\begin{aligned}
0 & =\frac{1}{r} \frac{\partial(r u)}{\partial r}+\frac{\partial w}{\partial z}, \\
\rho \dot{u}+\frac{\partial p}{\partial r} & =\frac{\partial}{\partial r}\left(\frac{\partial \mathcal{D}}{\partial u_{r}}\right)+\frac{\partial}{\partial z}\left(\frac{\partial \mathcal{D}}{\partial u_{z}}\right)-\phi_{r} \frac{\partial \mathcal{D}}{\partial \dot{\phi}}, \\
\rho \dot{w}+\frac{\partial p}{\partial z}= & \frac{\partial}{\partial r}\left(\frac{\partial \mathcal{D}}{\partial w_{r}}\right)+\frac{\partial}{\partial z}\left(\frac{\partial \mathcal{D}}{\partial w_{z}}\right)-\phi_{z} \frac{\partial \mathcal{D}}{\partial \dot{\phi}}, \\
0 & =\frac{\partial}{\partial r}\left(\frac{\partial \omega_{F}}{\partial \phi_{r}}\right)+\frac{\partial}{\partial z}\left(\frac{\partial \omega_{F}}{\partial \phi_{z}}\right)-\frac{\partial \omega_{F}}{\partial \phi}-\frac{\partial \mathcal{D}}{\partial \dot{\phi}},
\end{aligned}
$$

where the subscripts $r$ and $z$ in $\phi_{r}, \phi_{z}, u_{r}, u_{z}, w_{r}$, and $w_{z}$ represent the partial derivatives with respect to that variable, a superposed dot denotes the material time derivative, and $\rho$ is the constant fluid density. Two scalar quantities, the elastic free energy, $\omega_{F}$, and the dissipation function, $\mathcal{D}$, are also required, in order to close Eqs. (5)-(8). The elastic free energy describes the elastic energy associated with distortions to the director and the dissipation function describes the rate of viscous dissipation due to gradients in the velocity. We will use the Frank-Oseen free energy, $\omega_{F},{ }^{12}$ formulated using an assumption of small deformations and taking into account the symmetry of the nematic phase, given by

$$
\begin{aligned}
\omega_{F}= & \frac{1}{2} K_{1}(\nabla \cdot \mathbf{n})^{2}+\frac{1}{2} K_{2}(\mathbf{n} \cdot \nabla \times \mathbf{n})^{2}+\frac{1}{2} K_{3}(\mathbf{n} \times \nabla \times \mathbf{n})^{2} \\
& +\frac{1}{2}\left(K_{2}+K_{4}\right) \nabla \cdot((\mathbf{n} \cdot \nabla) \mathbf{n}-(\nabla \cdot \mathbf{n})),
\end{aligned}
$$

where the constants $K_{1}, K_{2}$, and $K_{3}$ are the nematic splay, twist, and bend elastic constants, and the combination $K_{2}+K_{4}$ is termed the saddle-splay elastic constant. To produce a mathematically tractable system of equations, it is common to assume that the splay, twist, and bend elastic constants are equal so that $K=K_{1}=K_{2}=K_{3}$ and that $K_{4}=0$. While this "one-constant approximation" is certainly a simplification of the material properties of a typical nematic, in practice, the ratios of elastic constants are rarely greater than two and the behavior is not expected to change qualitatively when this approximation is used. ${ }^{19}$ Using the one-constant approximation, the elastic free energy [Eq. (9)] becomes

$$
\omega_{F}=\frac{K}{2}\left[\left(\frac{\partial \phi}{\partial z}\right)^{2}+\left(\frac{\partial \phi}{\partial r}\right)^{2}+\frac{\cos ^{2} \phi}{r^{2}}\right] .
$$

For the dissipation function $\mathcal{D}$, we use the form proposed by Leslie ${ }^{16}$ given by

$$
\begin{aligned}
\mathcal{D}= & \frac{1}{2}\left[\alpha_{1}\left(n_{i} e_{i j} n_{j}\right)^{2}+2 \gamma_{2} N_{i} e_{i j} n_{j}+\alpha_{4} e_{i j} e_{i j}\right. \\
& \left.+\left(\alpha_{5}+\alpha_{6}\right) n_{i} e_{i j} e_{j k} n_{k}+\gamma_{1} N_{i} N_{i}\right],
\end{aligned}
$$

where $e_{i j}$ are the components of the rate of strain tensor, defined by $\mathbf{e}=\left(\nabla \mathbf{u}+(\nabla \mathbf{u})^{T}\right) / 2$, and the corotational time-flux $\mathbf{N}$ has components $N_{i}=\dot{n}_{i}-W_{i j} n_{j}$, where $W_{i j}$ are the components of the vorticity tensor, defined by $\mathbf{W}=\left(\nabla \mathbf{u}-(\nabla \mathbf{u})^{T}\right) / 2$. The parameters $\gamma_{1}=\alpha_{3}$ $-\alpha_{2}$ and $\gamma_{2}=\alpha_{3}+\alpha_{2}$ are the rotational and torsional viscosity coefficients, respectively, where $\alpha_{1}, \ldots, \alpha_{6}$ are the Leslie viscosities. ${ }^{12}$ Using Eqs. (2) and (3) leads to

$$
\begin{aligned}
\mathcal{D}= & \frac{1}{2}\left[\alpha_{1}\left(u_{r} \cos ^{2} \phi+\frac{1}{2}\left(u_{z}+w_{r}\right) \sin 2 \phi+w_{z} \sin ^{2} \phi\right)^{2}\right. \\
& +2 \gamma_{2}\left(\frac{1}{2}\left(w_{z}-u_{r}\right) \sin 2 \phi+\frac{1}{2}\left(u_{z}+w_{r}\right) \cos 2 \phi\right) \\
& \times\left(\dot{\phi}+\frac{1}{2}\left(u_{z}-w_{r}\right)\right)+\alpha_{4}\left(u_{r}^{2}+\frac{u^{2}}{r^{2}}+\frac{1}{2}\left(u_{z}+w_{r}\right)^{2}+w_{z}^{2}\right) \\
& +\left(\alpha_{5}+\alpha_{6}\right)\left(u_{r}^{2} \cos ^{2} \phi+\frac{1}{4}\left(u_{z}+w_{r}\right)^{2}+w_{z}^{2} \sin ^{2} \phi\right) \\
& \left.+\gamma_{1}\left(\dot{\phi}+\frac{1}{2}\left(u_{z}-w_{r}\right)\right)^{2}\right] .
\end{aligned}
$$




\section{B. Nondimensionalization}

In order to determine the important parameter groups and to enable progress in considering important asymptotic regimes, the equations are now nondimensionalized according to

$$
\begin{aligned}
& t=\frac{\mathcal{R}}{\mathcal{U}} \hat{t}, \quad r=\mathcal{R} \hat{r}, \quad z=\mathcal{H} \hat{z}, \\
& V=\mathcal{R}^{2} \mathcal{H} \hat{V}, \quad R(t)=\mathcal{R} \hat{R}(t), \quad h(t)=\mathcal{H} \hat{h}(t), \quad A=\mathcal{R}^{2} \hat{A}, \\
& \phi=\hat{\phi}, \quad u=\mathcal{U} \hat{u}, \quad w=\frac{\mathcal{U H}}{\mathcal{R}} \hat{w}, \quad p=\frac{\mu \mathcal{U} \mathcal{R}}{\mathcal{H}^{2}} \hat{p}, \\
& \gamma_{1}=\mu \hat{\gamma}_{1}, \quad \gamma_{2}=\mu \hat{\gamma}_{2}, \quad \alpha_{1}=\mu \hat{\alpha}_{1}, \quad \alpha_{2}=\mu \hat{\alpha}_{2}, \\
& \alpha_{3}=\mu \hat{\alpha}_{3}, \quad \alpha_{4}=\mu \hat{\alpha}_{4}, \quad \alpha_{5}=\mu \hat{\alpha}_{5}, \quad \alpha_{6}=\mu \hat{\alpha}_{6}, \\
& \omega_{F}=\frac{K}{\mathcal{H}^{2}} \hat{\omega}_{F}, \quad \mathcal{D}=\frac{\mu \mathcal{U}^{2}}{\mathcal{H}^{2}} \hat{\mathcal{D}}, \quad F=\frac{\mu \mathcal{U} \mathcal{R}^{3}}{\mathcal{H}^{2}} \hat{F}, \quad W_{p}=\frac{\mu \mathcal{U} \mathcal{R}^{3}}{\mathcal{H}^{2}} \hat{W}_{p},
\end{aligned}
$$

where the caret $\left(^{\wedge}\right)$ denotes nondimensional variables, $F$ is the force on the top plate, which will be introduced in Sec. III C, and $W_{p}$ is the weight of the top plate, which will be introduced in Sec. V. Typical values of the radial scale $\mathcal{R}$, height scale $\mathcal{H}$, and radial velocity scale $\mathcal{U}$ are given in Sec. II E. The pressure and viscosity are nondimensionalized using the isotropic viscosity, which is given by $\mu=\alpha_{4} / 2$ in terms of the Leslie viscosity $\alpha_{4}$, or, equivalently, by $\mu=\eta_{3}$ in terms of the Miesowicz viscosity $\eta_{3}$.

The nondimensional aspect ratio $\delta$, defined by the ratio of the height scale $\mathcal{H}$ and the radial scale $\mathcal{R}$, is

$$
\delta=\frac{\mathcal{H}}{\mathcal{R}} .
$$

In practice, the aspect ratio is typically small, corresponding to a thin film of nematic, for which the radial length scale is much larger than the height scale, and so a thin-film (i.e., small $\delta$ ) approximation is appropriate. The reduced Reynolds number, defined by

$$
\operatorname{Re}=\frac{\rho \mathcal{U} \mathcal{H}^{2}}{\mu \mathcal{R}},
$$

is a measure of the ratio of inertial forces and viscous forces within the system, and a large reduced Reynolds number corresponds to a system dominated by inertial effects, while a small reduced Reynolds number corresponds to a system dominated by viscous effects. The Ericksen number, defined by

$$
\operatorname{Er}=\frac{\mu \mathcal{U H}}{K}
$$

is a measure of the ratio of viscous effects and elasticity effects within the system, and a large Ericksen number corresponds to a system in which viscous effects are much stronger than elasticity effects, while a small Ericksen number corresponds to a system in which elasticity effects are much stronger than viscous effects.

From Eqs. (5)-(8), the nondimensional equations that govern the director angle $\phi$, the radial velocity component $u$, the vertical velocity component $w$, and the pressure $p$ are

$$
0=\frac{1}{r} \frac{\partial(r u)}{\partial r}+\frac{\partial w}{\partial z}
$$

$$
\begin{aligned}
\operatorname{Re} \rho \dot{u}+p_{r}= & \frac{\partial}{\partial r}\left[\alpha_{1}\left(\delta^{2} u_{r} \cos ^{4} \phi+\left(\delta u_{z}+\delta^{3} w_{r}\right) \sin \phi \cos ^{3} \phi+\delta^{2} w_{z} \sin ^{2} \phi \cos ^{2} \phi\right)-\frac{\gamma_{2}}{2}\left(\delta^{3} u_{r} \dot{\phi} \sin 2 \phi+\frac{1}{2}\left(\delta^{2} u_{z}-\delta^{4} w_{r}\right) u_{r} \sin 2 \phi\right)+2 \delta u_{r}\right. \\
& \left.+\left(\alpha_{5}+\alpha_{6}\right) \delta u_{r} \cos ^{2} \phi\right]+\frac{\partial}{\partial z}\left[\alpha_{1}\left(\delta u_{r} \sin \phi \cos ^{3} \phi+\left(u_{z}+\delta^{2} w_{r}\right) \sin ^{2} \phi \cos ^{2} \phi+\delta^{2} w_{z} \sin ^{3} \phi \cos \phi\right)\right. \\
& +\frac{\gamma_{2}}{2}\left(\delta \dot{\phi} \cos 2 \phi+\frac{1}{2}\left(u_{z}-\delta u_{r}\right) \cos 2 \phi+\frac{1}{2}\left(\delta w_{z}-\delta u_{r}\right) \sin 2 \phi+\frac{1}{2}\left(u_{z}+\delta^{2} w_{r}\right) \cos 2 \phi\right)+\left(u_{z}+\delta^{2} w_{r}\right)+\frac{\left(\alpha_{5}+\alpha_{6}\right)}{4}\left(u_{z}+\delta^{2} w_{r}\right) \\
& \left.+\frac{\gamma_{1}}{2}\left(\delta \dot{\phi}+\frac{1}{2}\left(u_{z}-\delta^{2} w_{r}\right)\right)\right]-\frac{\gamma_{2}}{2}\left(\left(\delta^{2} w_{z}-\delta^{2} u_{r}\right) \phi_{r} \sin 2 \phi+\left(\delta u_{z}+\delta^{3} w_{r}\right) \phi_{r} \cos 2 \phi\right)+\gamma_{1}\left(\delta^{2} \phi_{r} \dot{\phi}+\frac{1}{2}\left(\delta u_{z}-\delta^{3} w_{r}\right) \phi_{r}\right)
\end{aligned}
$$

$$
\begin{aligned}
\delta^{2} \operatorname{Re} \rho \dot{w}+p_{z}= & \frac{\partial}{\partial r}\left[\alpha_{1}\left(\delta^{3} u_{r} \sin \phi \cos ^{3} \phi+\frac{1}{2}\left(\delta^{2} u_{z}+\delta^{4} w_{r}\right) \sin ^{2} \phi \cos ^{2} \phi+\delta^{3} w_{z} \sin ^{3} \phi \cos \phi\right)-\frac{\gamma_{2}}{4}\left(\left(\delta^{3} w_{z}-\delta^{3} u_{r}\right) \sin 2 \phi\right.\right. \\
& \left.+\left(\delta^{2} u_{z}+\delta^{4} w_{r}\right) \cos 2 \phi\right)+\frac{\gamma_{2}}{2}\left(\delta^{3} \dot{\phi} \cos 2 \phi+\frac{1}{2}\left(\delta^{2} u_{z}-\delta^{4} w_{r}\right) \cos 2 \phi\right)+\frac{\alpha_{4}}{2}\left(\delta^{2} u_{z}+\delta^{4} w_{r}\right)+\frac{\left(\alpha_{5}+\alpha_{6}\right)}{4}\left(\delta^{2} u_{z}+\delta^{4} w_{r}\right) \\
& \left.-\frac{\gamma_{1}}{2}\left(\delta^{3} \dot{\phi}+\frac{1}{2}\left(\delta^{2} u_{z}-\delta^{4} w_{r}\right)\right)\right]+\frac{\partial}{\partial z}\left[\alpha_{1}\left(\delta^{2} u_{r} \sin ^{2} \phi \cos ^{2} \phi+\left(\delta u_{z}+\delta^{3} w_{r}\right) \sin ^{3} \phi \cos \phi+\delta^{2} w_{z} \sin ^{4} \phi\right)\right. \\
& \left.+\frac{\gamma_{2}}{2}\left(\delta^{2} \dot{\phi} \sin 2 \phi+\frac{1}{2}\left(\delta u_{z}-\delta^{3} w_{r}\right) \sin 2 \phi\right)+\alpha_{4} \delta^{2} w_{z}+\left(\alpha_{5}+\alpha_{6}\right) \delta^{2} w_{z} \sin ^{2} \phi\right] \\
& -\frac{\gamma_{2}}{2}\left(\left(\delta^{2} w_{z}-\delta^{2} u_{r}\right) \phi_{z} \sin 2 \phi+\left(\delta u_{z}+\delta^{3} w_{r}\right) \phi_{z} \cos 2 \phi\right)+\gamma_{1}\left(\delta^{2} \dot{\phi} \phi_{z}+\frac{1}{2}\left(\delta u_{z}-\delta^{3} w_{r}\right) \phi_{z}\right),
\end{aligned}
$$

and

$$
0=\phi_{z z}-\frac{1}{2} \operatorname{Er} u_{z}\left(\gamma_{1}+\gamma_{2} \cos 2 \phi\right)+\delta \operatorname{Er}\left[-\gamma_{2} \frac{1}{2}\left(w_{z}-u_{r}\right) \sin 2 \phi-\gamma_{1} \dot{\phi}\right]+\delta^{2}\left[\phi_{r r}-\frac{2 \sin 2 \phi}{r^{2}}-\frac{1}{2} \operatorname{Er} w_{r}\left(\gamma_{1}+\gamma_{2} \cos 2 \phi\right)\right]
$$


where we have now dropped the caret $\left(^{\wedge}\right)$ notation for simplicity since all quantities are now nondimensional. We will later solve Eqs. (17)-(20) using certain assumptions made about the size of the relative nondimensional parameters $\delta, \operatorname{Re}$, and Er and subject to appropriate boundary conditions on $\phi, u, w$, and $p$.

\section{Rescaled radial and vertical coordinates}

Before proceeding further, it is convenient to rescale the radial and vertical coordinates according to

$$
\tilde{r}=\frac{r}{R(t)}=\sqrt{\frac{\pi}{V}} h(t)^{1 / 2} r \quad \text { and } \quad \tilde{z}=\frac{z}{h(t)},
$$

where $\tilde{r}$ is the rescaled radial coordinate and $\tilde{z}$ is the rescaled vertical coordinate and where $\tilde{r}$ can be expressed in terms of the height of the top plate $h(t)$ using Eq. (1). In terms of the rescaled vertical coordinate $\tilde{z}$, the top plate and substrate are fixed at $\tilde{z}=1$ and $\tilde{z}=0$, respectively. The change in the height of the top plate $h(t)$ is included in the rescaled versions of the Ericksen-Leslie equations (17)-(20) via the appropriate rescaling of derivatives of $r$ and $z$, namely, $\partial / \partial r \rightarrow V^{-1 / 2} \pi^{1 / 2} h(t)^{1 / 2} \partial / \partial \tilde{r}$ and $\partial / \partial z \rightarrow h(t)^{-1} \partial / \partial \tilde{z}$, respectively.

Henceforth, we will express all equations and boundary conditions in terms of the rescaled coordinates $\tilde{r}$ and $\tilde{z}$ given by Eq. (21). However, for clarity and to aid physical interpretation, we will plot results in terms of the original unscaled radial coordinate $r$ and vertical coordinate $z$.

\section{Boundary conditions}

In order to solve Eqs. (17)-(20), appropriate boundary conditions must be imposed on the variables $\phi, u, w$, and $p$. For the velocity components, $u$ and $w$, we impose no-slip and no-penetration conditions on the solid boundaries at $\tilde{z}=0$ and $\tilde{z}=1$. The substrate at $\tilde{z}=0$ is stationary, and the top plate at $\tilde{z}=1$ is moving with vertical velocity $h^{\prime}(t)=\mathrm{d} h / \mathrm{d} t$, and so the appropriate boundary conditions are $u(\tilde{r}, 0, t)=0, u(\tilde{r}, 1, t)=0, w(\tilde{r}, 0, t)=0$, and $w(\tilde{r}, 1, t)=h^{\prime}(t)$. The pressure, $p$, is assumed to be fixed at the constant internal ambient pressure, $p_{\mathrm{I}}$, at the outer edge of the nematic drop so that $p(1, \tilde{z}, t)=p_{\text {I }}$. We impose regularity and axisymmetry at the center of the drop by assuming that $\partial p / \partial \tilde{r}=0$ at $\tilde{r}=0$. In summary, the boundary conditions for velocity and pressure are

$$
\begin{aligned}
u=0 & \text { on } \tilde{z}=0, \\
w=0 & \text { on } \tilde{z}=0, \\
u=0 & \text { on } \tilde{z}=1, \\
w=h^{\prime}(t) & \text { on } \tilde{z}=1,
\end{aligned}
$$

$$
\begin{array}{cc}
\frac{\partial p}{\partial \tilde{r}}=0 & \text { on } \tilde{r}=0 \\
p=p_{\mathrm{I}} & \text { on } \tilde{r}=1 .
\end{array}
$$

As mentioned previously, we consider four specific anchoring cases that commonly occur in nematic devices, namely, planar, homeotropic, hybrid aligned nematic (HAN), and $\pi$-cell anchoring. In the planar anchoring case, the director is parallel to the boundary at both the substrate and top plate so that $\phi(\tilde{r}, 0, t)=\phi(\tilde{r}, 1, t)=0$, and in the homeotropic anchoring case, the director is perpendicular to the boundary at both the substrate and top plate so that $\phi(\tilde{r}, 0, t)=\phi(\tilde{r}, 1, t)=\pi / 2$. In the HAN anchoring case, the director is parallel to the substrate and perpendicular to the top plate so that $\phi(\tilde{r}, 0, t)=0$ and $\phi(\tilde{r}, 1, t)=\pi / 2$, and in the $\pi$-cell anchoring case, the director is parallel to the substrate so that $\phi(\tilde{r}, 0, t)=0$ and parallel to the top plate so that $\phi(\tilde{r}, 1, t)=\pi$. The equilibrium director configurations in the limit of no flow for these anchoring cases, namely, Eqs. (53)-(56), will be calculated later and are sketched in Fig. 2.

It is worth noting at this point that the axisymmetric form of the nondimensional elastic energy given by Eq. (10) is

$$
\omega_{F}=\frac{1}{2}\left[\frac{1}{h(t)^{2}}\left(\frac{\partial \phi}{\partial \tilde{z}}\right)^{2}+\delta^{2} \frac{\pi}{V} h(t)\left(\left(\frac{\partial \phi}{\partial \tilde{r}}\right)^{2}+\frac{\cos ^{2} \phi}{\tilde{r}^{2}}\right)\right],
$$

and therefore, the elastic energy is undefined if $\phi \neq \pi / 2$ at $\tilde{r}=0$, a situation that occurs for the planar, HAN, and $\pi$-cell anchoring cases. This type of singularity in the elastic energy is associated with a discontinuity in the director orientation and can lead to point or line defects. At such defects, a disordering transition occurs and a director description of the nematic is not valid. ${ }^{12,14}$ However, in Secs. II E and II F, we will find that since the aspect ratio is small (i.e., $\delta \ll 1$ ), the singular $\tilde{r}^{-2}$ term in Eq. (28) does not appear at leading order in $\delta$. The results in this work are therefore valid away from the center of the drop at $\tilde{r}=0$. In summary, the boundary conditions for the four anchoring cases are

$$
\begin{aligned}
\text { planar: } & \phi=0 \text { on } \tilde{z}=0, \phi=0 \text { on } \tilde{z}=1, \\
\text { homeotropic: } & \phi=\pi / 2 \text { on } \tilde{z}=0, \phi=\pi / 2 \text { on } \tilde{z}=1, \\
\text { HAN: } & \phi=0 \text { on } \tilde{z}=0, \phi=\pi / 2 \text { on } \tilde{z}=1, \\
\pi \text {-cell: } & \phi=0 \text { on } \tilde{z}=0, \phi=\pi \text { on } \tilde{z}=1 .
\end{aligned}
$$

\section{E. Typical values of nondimensional groups}

In this subsection, we consider the asymptotic regimes for the sizes of the nondimensional groups $\delta, \mathrm{Re}$, and $\mathrm{Er}$ corresponding to

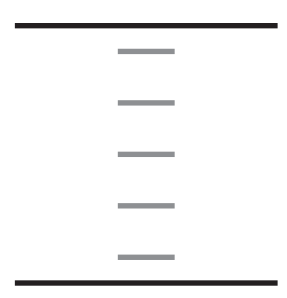

(a) planar

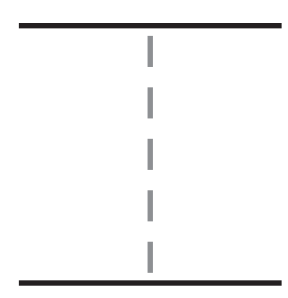

(b) homeotropic

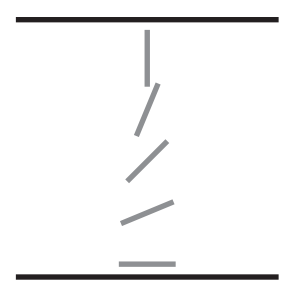

(c) HAN

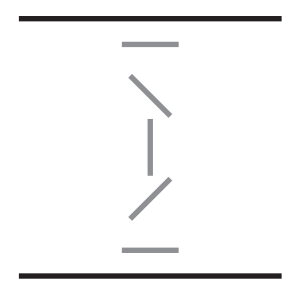

(d) $\pi$-cell
FIG. 2. Sketches of the equilibrium director configurations in the limit of no flow for the four specific anchoring cases considered: (a) planar, (b) homeotropic, (c) $\mathrm{HAN}$, and (d) $\pi$-cell anchoring. 
TABLE I. Typical values of the nondimensional groups in current manufacturing processes using the ODF method, calculated using the following typical parameter values: height of the drop $\mathcal{H}=50 \mu \mathrm{m}$, radius of the drop $\mathcal{R}=5 \mathrm{~mm}$, typical top plate speed $w_{p}=1 \mathrm{~mm} \mathrm{~s}^{-1}$, isotropic viscosity $\mu=0.01 \mathrm{~Pa} \mathrm{~s}$, one-constant elastic constant $K=10 \mathrm{pN}$, and density $\rho=1000 \mathrm{~kg} \mathrm{~m}^{-3}$. 8,19

\begin{tabular}{lcc}
\hline \hline Nondimensional group & Definition & Typical value \\
\hline$\delta$ & $\mathcal{H} / \mathcal{R}$ & $10^{-2}$ \\
$\operatorname{Re}$ & $\rho \mathcal{U} \mathcal{H}^{2} / \mu \mathcal{R}=\rho w_{p} \mathcal{H}^{2} / \mu$ & $5 \times 10^{-3}$ \\
$\mathrm{Er}$ & $\mu \mathcal{U H} / K=\mu w_{p} \mathcal{R} / K$ & $5 \times 10^{3}$ \\
\hline \hline
\end{tabular}

typical length scales and plate speeds that occur in both the current and possible future manufacturing regimes using the ODF method.

In current manufacturing processes using the ODF method, typical parameter values are height of the drop $\mathcal{H}=50 \mu \mathrm{m}$, radius of the film $\mathcal{R}=5 \mathrm{~mm}$, vertical velocity (i.e., a typical top plate speed) $w_{p}=1 \mathrm{~mm} \mathrm{~s}^{-1}$, isotropic viscosity $\mu=0.01 \mathrm{~Pa} \mathrm{~s}$, one-constant elastic constant $K=10 \mathrm{pN}$, and density $\rho=1000 \mathrm{~kg} \mathrm{~m}^{-3}$. 8,19 The radial velocity scale, $\mathcal{U}$, appearing in the reduced Reynolds number and Ericksen number is calculated using the conservation of mass equation (17) to give $\mathcal{U}=w_{p} \mathcal{R} / \mathcal{H}$. As Table I shows, the assumptions of small aspect ratio $(\delta \ll 1)$ and small reduced Reynolds number (Re $\ll 1)$ are well justified for these parameter values. The Ericksen number is typically much larger than unity during current manufacturing processes, indicating that viscous effects are typically stronger than elasticity effects and that a large Ericksen number approximation, Er $\gg 1$, is appropriate.

However, as described earlier, in this work, we consider the possibility of using smaller drops of nematic and slower top plate speeds in order to access a different possible future manufacturing regime. Specifically, we consider typical values for ink-jet printed drops, ${ }^{21,22}$ where the length scales are reduced by a factor of 100 , giving a height of the drop $\mathcal{H}=0.5 \mu \mathrm{m}$, a radius of the $\operatorname{drop} \mathcal{R}=50 \mu \mathrm{m}$, and a reduced top plate speed of $w_{p}=2 \mu \mathrm{m} \mathrm{s}^{-1}$. All of the material parameters (namely, the viscosity, elastic constant, and density of the nematic) remain the same as before. As Table II shows, using these values, the assumptions of small aspect ratio $(\delta \ll 1)$ and small reduced Reynolds number $(\mathrm{Re} \ll 1)$ remain well justified, but now the value of the Ericksen number is smaller, indicating that a small Ericksen number approximation, $\mathrm{Er} \ll 1$, is now appropriate. In this work, we will therefore consider the asymptotic regime in which $\delta \ll 1, \operatorname{Re} \ll 1$, and $\operatorname{Er} \ll 1$, which corresponds to a thin film of

TABLE II. Typical values of the nondimensional groups in possible future manufacturing processes using the ODF method, calculated using the following typical parameter values: height of the drop $\mathcal{H}=0.5 \mu \mathrm{m}$, radius of the drop $\mathcal{R}=50 \mu \mathrm{m}$, a reduced top plate speed $w_{p}=2 \mu \mathrm{m} \mathrm{s}^{-1}$, isotropic viscosity $\mu=0.01 \mathrm{~Pa} \mathrm{~s}$, one-constant elastic constant $K=10 \mathrm{pN}$, and density $\rho=1000 \mathrm{~kg} \mathrm{~m}^{-3} \cdot{ }^{19}$

\begin{tabular}{lcc}
\hline \hline Nondimensional group & Definition & Typical value \\
\hline$\delta$ & $\mathcal{H} / \mathcal{R}$ & $10^{-2}$ \\
$\operatorname{Re}$ & $\rho \mathcal{U H} \mathcal{H}^{2} / \mu \mathcal{R}=\rho w_{p} \mathcal{H}^{2} / \mu$ & $10^{-7}$ \\
$\mathrm{Er}$ & $\mu \mathcal{U H} / K=\mu w_{p} \mathcal{R} / K$ & $10^{-1}$ \\
\hline \hline
\end{tabular}

nematic in which inertial effects are weak and elasticity effects are strong compared to viscous effects. Similar regimes for the flow of nematic have been considered for a number of classical problems in fluid dynamics including flow down an inclined plane and channel flows (see, for example, Refs. 23-27).

\section{F. The thin-film approximation}

With the assumptions that the aspect ratio and the Reynolds number are both small, $\delta \ll 1$ and $\operatorname{Re} \ll 1$, at leading order in $\delta$ the radial momentum equation (18), rescaled using Eq. (21), becomes

$$
\begin{aligned}
\frac{\partial p}{\partial \tilde{r}}= & \sqrt{\frac{V}{\pi}} h(t)^{-5 / 2} \frac{\partial}{\partial \tilde{z}}\left[\left(\frac{\alpha_{1}}{4} \sin ^{2} 2 \phi+\frac{\gamma_{2}}{2} \cos 2 \phi+1\right.\right. \\
& \left.\left.+\frac{\alpha_{5}+\alpha_{6}}{4}+\frac{\gamma_{1}}{4}\right) \frac{\partial u}{\partial \tilde{z}}\right] .
\end{aligned}
$$

With the Parodi relation, $\alpha_{6}=\alpha_{2}+\alpha_{3}+\alpha_{5}{ }^{12}$ we can rewrite Eq. (33) in terms of the nondimensional Miesowicz viscosities, ${ }^{12}$ defined by

$$
\eta_{12}=\alpha_{1}, \quad \eta_{1}=\frac{1}{2}\left(\alpha_{2}+2 \alpha_{3}+2+\alpha_{5}\right), \quad \eta_{2}=\frac{1}{2}\left(-\alpha_{2}+2+\alpha_{5}\right)
$$

as

$$
\begin{aligned}
\frac{\partial p}{\partial \tilde{r}}= & \sqrt{\frac{V}{\pi}} h(t)^{-5 / 2} \frac{\partial}{\partial \tilde{z}}\left[\left(\eta_{12} \sin ^{2} \phi \cos ^{2} \phi+\eta_{1} \cos ^{2} \phi\right.\right. \\
& \left.\left.+\eta_{2} \sin ^{2} \phi\right) \frac{\partial u}{\partial \tilde{z}}\right] .
\end{aligned}
$$

Similarly the vertical momentum equation, (19), rescaled using Eq. (21), becomes

$$
\frac{\partial p}{\partial \tilde{z}}=0
$$

Therefore, at leading order in $\delta$ the pressure is independent of the rescaled vertical coordinate $\tilde{z}$ and hence is given by

$$
p=p(\tilde{r}, t) \text {. }
$$

In addition, the angular momentum equation (20), again rescaled using Eq. (21), becomes

$$
0=\frac{1}{h(t)} \frac{\partial^{2} \phi}{\partial \tilde{z}^{2}}-\frac{1}{2} \operatorname{Er}\left(\gamma_{1}+\gamma_{2} \cos 2 \phi\right) \frac{\partial u}{\partial \tilde{z}} .
$$

In summary, in what follows we will solve Eqs. (17), (35), and (38) subject to the boundary conditions [Eqs. (22)-(27)] together with the appropriate conditions from Eqs. (29)-(32) for the particular problem under consideration for the dependent variables $\phi(\tilde{r}, \tilde{z}, t)$, $u(\tilde{r}, \tilde{z}, t), w(\tilde{r}, \tilde{z}, t)$, and $p(\tilde{r}, t)$.

\section{THE LIMIT OF SMALL ERICKSEN NUMBER}

In the limit of small Ericksen number $\mathrm{Er} \ll 1$, we seek an asymptotic solution to the problem in the form

$$
\begin{aligned}
\phi & =\phi_{0}+\operatorname{Er} \phi_{1}+\operatorname{Er}^{2} \phi_{2}+\mathcal{O}\left(\operatorname{Er}^{3}\right), \\
u & =u_{0}+\operatorname{Er} u_{1}+\operatorname{Er}^{2} u_{2}+\mathcal{O}\left(\operatorname{Er}^{3}\right), \\
w & =w_{0}+\operatorname{Er} w_{1}+\operatorname{Er}^{2} w_{2}+\mathcal{O}\left(\operatorname{Er}^{3}\right), \\
p & =p_{0}+\operatorname{Er} p_{1}+\operatorname{Er}^{2} p_{2}+\mathcal{O}\left(\operatorname{Er}^{3}\right) .
\end{aligned}
$$


Substituting these asymptotic expansions into the governing equations (17), (35), and (38), we are able to obtain the leading-order solutions that describe the dominant behavior at small Ericksen number. By finding the higher-order corrections to these solutions, we are then able to describe the perturbations to this leading-order behavior. Using the expansions for the velocity components given by Eqs. (40) and (41) and the rescaling equation (21), the conservation of mass equation (17) takes the same form at each order in the Ericksen number, namely,

$$
\sqrt{\frac{\pi}{V}} h(t)^{3 / 2} \frac{1}{\tilde{r}} \frac{\partial\left(\tilde{r} u_{i}\right)}{\partial \tilde{r}}+\frac{\partial w_{i}}{\partial \tilde{z}}=0
$$

for $i=0,1,2, \ldots$ Using the expansions (39)-(42) and the rescaling equation (21) in Eqs. (35) and (38), and considering the appropriate expressions at different orders in the Ericksen number, yields, at leading order,

$$
\begin{aligned}
\sqrt{\frac{\pi}{V}} h(t)^{5 / 2} \frac{\partial p_{0}}{\partial \tilde{r}} & =\frac{\partial}{\partial \tilde{z}}\left[g\left(\phi_{0}\right) \frac{\partial u_{0}}{\partial \tilde{z}}\right], \\
\frac{\partial^{2} \phi_{0}}{\partial \tilde{z}^{2}} & =0
\end{aligned}
$$

at first order,

$$
\begin{aligned}
\sqrt{\frac{\pi}{V}} h(t)^{5 / 2} \frac{\partial p_{1}}{\partial \tilde{r}} & =\frac{\partial}{\partial \tilde{z}}\left[g^{\prime}\left(\phi_{0}\right) \phi_{1} \frac{\partial u_{0}}{\partial \tilde{z}}+g\left(\phi_{0}\right) \frac{\partial u_{1}}{\partial \tilde{z}}\right], \\
\frac{1}{h(t)} \frac{\partial^{2} \phi_{1}}{\partial \tilde{z}^{2}} & =m\left(\phi_{0}\right) \frac{\partial u_{0}}{\partial \tilde{z}}
\end{aligned}
$$

and at second order,

$$
\begin{aligned}
\sqrt{\frac{\pi}{V}} h(t)^{5 / 2} \frac{\partial p_{2}}{\partial \tilde{r}}= & \frac{\partial}{\partial \tilde{z}}\left[g^{\prime}\left(\phi_{0}\right) \phi_{2} \frac{\partial u_{0}}{\partial \tilde{z}}+\frac{1}{2} g^{\prime \prime}\left(\phi_{0}\right) \phi_{1}^{2} \frac{\partial u_{0}}{\partial \tilde{z}}\right. \\
& \left.+g^{\prime}\left(\phi_{0}\right) \phi_{1} \frac{\partial u_{1}}{\partial \tilde{z}}+g\left(\phi_{0}\right) \frac{\partial u_{2}}{\partial \tilde{z}}\right] \\
\frac{1}{h(t)} \frac{\partial^{2} \phi_{2}}{\partial \tilde{z}^{2}}= & m^{\prime}\left(\phi_{0}\right) \phi_{1} \frac{\partial u_{0}}{\partial \tilde{z}}+m\left(\phi_{0}\right) \frac{\partial u_{1}}{\partial \tilde{z}}
\end{aligned}
$$

where

$$
\begin{aligned}
g(\phi) & =\eta_{12} \sin ^{2} \phi \cos ^{2} \phi+\eta_{1} \cos ^{2} \phi+\eta_{2} \sin ^{2} \phi, \\
m(\phi) & =\gamma_{1}+\gamma_{2} \cos 2 \phi .
\end{aligned}
$$

Equations (45) and (47) show that while the flow of the nematic does not affect the leading-order director angle $\phi_{0}$, the leading-order radial flow $u_{0}$ may affect the first-order director angle $\phi_{1}$. Indeed, Eq. (47) has similarities to that describing the classical flow alignment problem in a nematic ${ }^{12}$ and, as we will see later, has similar behavior. Specifically, when the leading-order shear rate $\partial u_{0} / \partial \tilde{z}$ is large, the director will be forced to align, at least away from boundaries, at angles given by $m\left(\phi_{0}\right)=0$. For a positive shear rate, the relevant solution to $m\left(\phi_{0}\right)=0$ is

$$
\phi_{0}=\phi_{\mathrm{L}}=\frac{1}{2} \cos ^{-1}\left(-\frac{\gamma_{1}}{\gamma_{2}}\right),
$$

where $\phi_{\mathrm{L}}$ is the Leslie angle, ${ }^{12}$ while for negative shear rates, the director angle will tend toward $\phi_{0}=-\phi_{\mathrm{L}}$. Since the director is invariant to rotations of $\pi$, there are an infinite number of "positive"
Leslie angles, $k \pi+\phi_{\mathrm{L}}$, and "negative" Leslie angles, $k \pi-\phi_{\mathrm{L}}$, for any integer $k$. We note that the terms "positive" and "negative" Leslie angles refer to the sign of the shear rate that is flow aligning the director rather than to the sign of the numerical value of the angle. Considering various different Leslie angles will be important in Sec. IV D for understanding the behavior of the first-order director angle $\phi_{1}$ in each of the four anchoring cases.

\section{A. General solution}

The leading-order angular momentum equation (45) can be immediately solved to yield the leading-order director angle $\phi_{0}$ $=\phi_{0}(\tilde{z})$ for each of the four anchoring cases given by Eqs. (29)-(32), namely,

$$
\begin{aligned}
& \phi_{0}=0 \text { for the planar anchoring case, } \\
& \phi_{0}=\frac{\pi}{2} \text { for the homeotropic anchoring case, } \\
& \phi_{0}=\frac{\pi \tilde{z}}{2} \text { for the HAN anchoring case, } \\
& \phi_{0}=\pi \tilde{z} \text { for the } \pi \text {-cell anchoring case. }
\end{aligned}
$$

These solutions are the equilibrium director configurations in the limit of no flow for the four anchoring cases previously sketched in Fig. 2. Note that these solutions for $\phi_{0}$ given in Eqs. (55) and (56) are dependent on time via the rescaled vertical coordinate $\tilde{z}$. Integrating Eq. (44) twice with respect to $\tilde{z}$ and using the boundary conditions [Eqs. (22) and (24)] then yields the solution for the leading-order radial velocity,

$$
u_{0}(\tilde{r}, \tilde{z}, t)=\sqrt{\frac{\pi}{V}} h(t)^{5 / 2} \int_{0}^{\tilde{z}} \frac{1}{g\left(\phi_{0}\right)}\left(\frac{\partial p_{0}(\tilde{r}, t)}{\partial \tilde{r}} \tilde{z}+C\right) \mathrm{d} \xi
$$

where the function $C=C(\tilde{r}, t)$ is found from the boundary conditions to be

$$
C=-\frac{\partial p_{0}(\tilde{r}, t)}{\partial \tilde{r}} \int_{0}^{1} \frac{\tilde{z}}{g\left(\phi_{0}\right)} \mathrm{d} \tilde{z}\left(\int_{0}^{1} \frac{\mathrm{d} \tilde{z}}{g\left(\phi_{0}\right)}\right)^{-1} .
$$

From Eqs. (22), (57), and (58), we can then express the leading-order radial velocity as

$$
u_{0}(\tilde{r}, \tilde{z}, t)=\sqrt{\frac{\pi}{V}} h(t)^{5 / 2} \frac{\partial p_{0}(\tilde{r}, t)}{\partial r} \Pi_{1}(\tilde{z}),
$$

where

$$
\begin{aligned}
\Pi_{1}(\tilde{z})= & \int_{0}^{\tilde{z}} \frac{\xi}{g\left(\phi_{0}\right)} \mathrm{d} \xi-\int_{0}^{\tilde{z}} \frac{1}{g\left(\phi_{0}\right)} \mathrm{d} \xi \int_{0}^{1} \frac{\tilde{z}}{g\left(\phi_{0}\right)} \mathrm{d} \tilde{z} \\
& \times\left(\int_{0}^{1} \frac{1}{g\left(\phi_{0}\right)} \mathrm{d} \tilde{z}\right)^{-1} .
\end{aligned}
$$

The first-order correction to the leading-order director angle, $\phi_{1}$, is calculated by integrating the first-order angular momentum equation (47), twice with respect to $\tilde{z}$, and using the solution for $u_{0}$ given by Eq. (59) and the boundary conditions $\phi_{1}=0$ on both $\tilde{z}=0$ and $\tilde{z}=1$, to give

$$
\phi_{1}(\tilde{r}, \tilde{z}, t)=\sqrt{\frac{\pi}{V}} h(t)^{7 / 2} \frac{\partial p_{0}(\tilde{r}, t)}{\partial \tilde{r}} \Pi_{2}(\tilde{z}),
$$


where

$$
\Pi_{2}(\tilde{z})=\int_{0}^{\tilde{z}} \int_{0}^{\xi} m\left(\phi_{0}\right) \frac{\mathrm{d} \Pi_{1}}{\mathrm{~d} \zeta} \mathrm{d} \zeta \mathrm{d} \xi-\tilde{z} \int_{0}^{1} \int_{0}^{\zeta} m\left(\phi_{0}\right) \frac{\mathrm{d} \Pi_{1}}{\mathrm{~d} \xi} \mathrm{d} \xi \mathrm{d} \zeta .
$$

We can now calculate the first-order correction to the leading-order radial velocity, $u_{1}$, by integrating Eq. (46) with respect to $z$, using expressions for $u_{0}$ and $\phi_{1}$ from Eqs. (59) and (61) as well as the firstorder boundary conditions $u_{1}=0$ on both $\tilde{z}=0$ and $\tilde{z}=1$, to obtain

$$
\begin{aligned}
u_{1}(\tilde{r}, \tilde{z}, t)= & \sqrt{\frac{\pi}{V}} h(t)^{5 / 2} \frac{\partial p_{1}(\tilde{r}, t)}{\partial \tilde{r}} \Pi_{1}(\tilde{z}) \\
& +\frac{\pi}{V} h(t)^{6}\left(\frac{\partial p_{0}(\tilde{r}, t)}{\partial \tilde{r}}\right)^{2} \Pi_{3}(\tilde{z}),
\end{aligned}
$$

where

$$
\begin{aligned}
\Pi_{3}(\tilde{z})= & \int_{0}^{\tilde{z}} \frac{1}{g\left(\phi_{0}\right)} \mathrm{d} \xi\left(\int_{0}^{1} \frac{1}{g\left(\phi_{0}\right)} \mathrm{d} \zeta\right)^{-1} \int_{0}^{1} \frac{g^{\prime}\left(\phi_{0}\right) \Pi_{2} \frac{\mathrm{d} \Pi_{1}}{\mathrm{~d} \zeta}}{g\left(\phi_{0}\right)} \mathrm{d} \zeta \\
& -\int_{0}^{\tilde{z}} \frac{g^{\prime}\left(\phi_{0}\right) \Pi_{2} \frac{\mathrm{d} \Pi_{1}}{\mathrm{~d} \xi}}{g\left(\phi_{0}\right)} \mathrm{d} \xi .
\end{aligned}
$$

To calculate the leading- and first-order vertical velocity components, $w_{0}$ and $w_{1}$, the solutions for $u_{0}$ and $u_{1}$, given by Eqs. (59) and (63), and the conservation of mass equation (43), with $i=0,1$, are used, yielding

$$
\begin{aligned}
w_{0}(\tilde{r}, \tilde{z}, t)= & -\frac{\pi}{V} h(t) \frac{1}{\tilde{r}} \frac{\partial}{\partial \tilde{r}}\left(\tilde{r} \frac{\partial p_{0}(\tilde{r}, t)}{\partial \tilde{r}}\right) \int_{0}^{\tilde{z}} \Pi_{1}(\xi) \mathrm{d} \xi \\
w_{1}(\tilde{r}, \tilde{z}, t)= & -\frac{\pi}{V} h(t) \frac{1}{\tilde{r}} \frac{\partial}{\partial \tilde{r}}\left(\tilde{r} \frac{\partial p_{1}(\tilde{r}, t)}{\partial \tilde{r}}\right) \int_{0}^{\tilde{z}} \Pi_{1}(\xi) \mathrm{d} \xi-\left(\frac{\pi}{V}\right)^{3 / 2} \\
& \times h(t)^{15 / 2} \frac{1}{\tilde{r}} \frac{\partial}{\partial \tilde{r}}\left(\tilde{r}\left(\frac{\partial p_{0}(\tilde{r}, t)}{\partial \tilde{r}}\right)^{2}\right) \int_{0}^{\tilde{z}} \Pi_{3}(\xi) \mathrm{d} \xi
\end{aligned}
$$

To calculate the leading-order pressure, $p_{0}$, we apply the boundary condition [Eq. (25)] to the leading-order vertical velocity, given by Eq. (65), and integrate with respect to $\tilde{r}$ and impose the condition on the pressure gradient [Eq. (26)] which leads to

$$
\frac{\partial p_{0}(\tilde{r}, t)}{\partial \tilde{r}}=-\frac{V h^{\prime}(t) \tilde{r}}{2 \pi h(t)^{4}}\left(\int_{0}^{1} \Pi_{1}(\xi) \mathrm{d} \xi\right)^{-1} .
$$

The leading-order pressure gradient [Eq. (67)] can be substituted into Eqs. (59), (61), (63), (65), and (66) to yield the full solutions for $\phi_{1}, u_{0}, u_{1}, w_{0}$, and $w_{1}$, respectively. A further integration of Eq. (67) with respect to $\tilde{r}$ and application of the boundary condition on the pressure [Eq. (27)] yields the solution for the leading-order pressure,

$$
p_{0}(\tilde{r}, t)=p_{\text {I }}+\frac{V h^{\prime}(t)}{4 \pi h(t)^{4}}\left(1-\tilde{r}^{2}\right)\left(\int_{0}^{1} \Pi_{1}(\xi) \mathrm{d} \xi\right)^{-1} .
$$

The same approach is used to calculate the first-order pressure gradient and the first-order pressure,

$$
\begin{aligned}
\frac{\partial p_{1}(\tilde{r}, t)}{\partial r} & =-\frac{V^{3 / 2} h^{\prime}(t)^{2}}{4 \pi^{3 / 2} h(t)^{9 / 2}} \tilde{r}^{2}\left(\int_{0}^{1} \Pi_{1}(\xi) \mathrm{d} \xi\right)^{-3} \int_{0}^{1} \Pi_{3}(\xi) \mathrm{d} \xi, \quad(69) \\
p_{1}(\tilde{r}, t) & =\frac{V^{3 / 2} h^{\prime}(t)^{2}}{12 \pi^{3 / 2} h(t)^{9 / 2}}\left(1-\tilde{r}^{3}\right)\left(\int_{0}^{1} \Pi_{1}(\xi) \mathrm{d} \xi\right)^{-3} \int_{0}^{1} \Pi_{3}(\xi) \mathrm{d} \xi .
\end{aligned}
$$

In summary, in addition to the solutions for the leading-order director angle $\phi_{0}$ for each anchoring case, given by Eqs. (53)-(56), we find the leading-order radial velocity $u_{0}$, given by Eq. (59), the firstorder director angle $\phi_{1}$, given by Eq. (61), first-order radial velocity $u_{1}$, given by Eq. (63), leading-order vertical velocity $w_{0}$, given by Eq. (65), first-order vertical velocity $w_{1}$, given by Eq. (66), leadingorder pressure $p_{0}$, given by Eq. (68), and first-order pressure $p_{1}$, given by Eq. (70). Equations (59), (61), (63), (65), (66), (68), and (70) describe the director angle, velocity, and pressure at leading and first order for any fixed angles of the director at the substrate and the top plate; however, in this work, we will focus on the four anchoring cases given by Eqs. (29)-(32). In fact, Eqs. (59), (61), (63), (65), (66), (68), and (70) provide the solution at first-order for any leading-order director angle $\phi_{0}$, including the more general situation where one or more of the plates exhibit weak anchoring ${ }^{28}$ rather than infinite anchoring. A weak anchoring condition is a Robin condition for the director angle, for instance, as in the Rapini-Papoular form $K \partial \phi / \partial z \pm \mathcal{A} \sin 2 \phi=0,{ }^{28}$ where $\mathcal{A}$ is known as the anchoring strength.

The leading-order pressure, given by Eq. (68), can be expressed as

$$
p_{0}=p_{\mathrm{I}}-\eta\left(\phi_{0}\right) \frac{3 V h^{\prime}(t)}{\pi h(t)^{4}}\left(1-\tilde{r}^{2}\right)
$$

where

$$
\eta\left(\phi_{0}\right)=-\left(12 \int_{0}^{1} \Pi_{1}(\xi) \mathrm{d} \xi\right)^{-1}
$$

is an effective viscosity which depends on the leading-order director angle via the expression for $\Pi_{1}$ given by Eq. (60). For future reference, the values of the effective viscosity $\eta\left(\phi_{0}\right)$ for the four anchoring cases given by Eqs. (29)-(32), are listed in Table III using parameter values for the standard nematic 4-Cyano-4'-pentylbiphenyl (5CB). ${ }^{12}$ Table III shows that $\eta\left(\phi_{0}\right)$ is largest for the homeotropic anchoring case for which $\eta\left(\phi_{0}\right)=\eta_{2}$ and smallest for the planar anchoring case for which $\eta\left(\phi_{0}\right)=\eta_{1}$.

Note that substituting Eq. (71) into Eqs. (59), (61), and (65) yields

$$
\begin{aligned}
\phi_{1}(\tilde{r}, \tilde{z}, t) & =6 \sqrt{\frac{V}{\pi}} \frac{\eta\left(\phi_{0}\right) h^{\prime}(t)}{h(t)^{1 / 2}} \Pi_{2}(\tilde{z}) \tilde{r}, \\
u_{0}(\tilde{r}, \tilde{z}, t) & =6 \sqrt{\frac{V}{\pi}} \frac{\eta\left(\phi_{0}\right) h^{\prime}(t)}{h(t)^{3 / 2}} \Pi_{1}(\tilde{z}) \tilde{r}, \\
w_{0}(\tilde{z}, t) & =12 \eta\left(\phi_{0}\right) h^{\prime}(t) \int_{0}^{\tilde{z}} \Pi_{1}(\xi) \mathrm{d} \xi,
\end{aligned}
$$

TABLE III. Values of the effective viscosity $\eta\left(\phi_{0}\right)$ given by Eq. (72) for the four anchoring cases given by Eqs. (29)-(32) using the material parameter values for the nematic $5 \mathrm{CB} .{ }^{12}$

\begin{tabular}{lc}
\hline \hline Anchoring case & Effective viscosity $\eta\left(\phi_{0}\right)$ \\
\hline Planar & $\eta_{1}=0.6258$ \\
Homeotropic & $\eta_{2}=3.2270$ \\
HAN & 1.6006 \\
$\pi$-cell & 0.9184 \\
\hline
\end{tabular}


and if we substitute $\eta\left(\phi_{0}\right)=1$ and $g\left(\phi_{0}\right)=1$ into Eqs. (71), (74), and (75), we recover the classical solution to the Newtonian squeeze-film problem.

Equations (73)-(75) show that the first-order director angle and the leading-order radial velocity are proportional to $\tilde{r}$, while the leading-order vertical velocity is independent of $\tilde{r}$. Thus, the magnitudes, but not the qualitative behavior, of $\phi_{1}$ and $u_{0}$ vary with $\tilde{r}$. Equations (68) and (73)-(75) also give the time dependence of the solutions $\phi_{1}, u_{0}, w_{0}$, and $p_{0}$, namely,

$\phi_{1} \propto \frac{h^{\prime}(t)}{h(t)^{1 / 2}}, \quad u_{0} \propto \frac{h^{\prime}(t)}{h(t)^{3 / 2}}, \quad w_{0} \propto h^{\prime}(t), \quad$ and $\quad p_{0} \propto \frac{h^{\prime}(t)}{h(t)^{4}}$.

While Eqs. (59), (61), (63), (65), (66), (68), and (70) provide explicit expressions for the leading- and first-order solutions in the limit of small Ericksen number, these expressions depend on the calculation of the integrals $\Pi_{1}, \Pi_{2}$, and $\Pi_{3}$. These integrals cannot be analytically evaluated for general forms of the leading-order director angle $\phi_{0}$. However, in two of the four anchoring cases, we consider, namely, the planar [Eq. (29)] and homeotropic [Eq. (30)] anchoring cases, $g\left(\phi_{0}\right)$ is constant and hence further analytic progress is possible. For the other two anchoring cases, namely, the HAN [Eq. (31)] and $\pi$-cell [Eq. (32)] anchoring cases, $g\left(\phi_{0}\right)$ is not constant, and so the integrals and solutions must, in general, be evaluated numerically.

For the two cases in which $\phi_{0}$ is constant, and so $\eta\left(\phi_{0}\right), g\left(\phi_{0}\right)$, and $m\left(\phi_{0}\right)$ are constant and $\eta\left(\phi_{0}\right)=g\left(\phi_{0}\right)$, analytical expressions for the integrals $\Pi_{1}, \Pi_{2}$, and $\Pi_{3}$ can be readily obtained and used to calculate the leading- and first-order solutions, namely,

$$
\begin{gathered}
\phi_{1}=\frac{1}{2} \sqrt{\frac{V}{\pi}} \frac{m\left(\phi_{0}\right) h^{\prime}(t)}{h(t)^{1 / 2}} \tilde{z}(1-2 \tilde{z})(1-\tilde{z}) \tilde{r} \\
u_{0}=-3 \sqrt{\frac{V}{\pi}} \frac{h^{\prime}(t)}{h(t)^{3 / 2}} \tilde{z}(1-\tilde{z}) \tilde{r} \\
u_{1}=0 \\
w_{0}=h^{\prime}(t) \tilde{z}^{2}(3-2 \tilde{z}), \\
w_{1}=0 \\
p_{0}=p_{\mathrm{I}}-\frac{3 g\left(\phi_{0}\right) V h^{\prime}(t)}{\pi h(t)^{4}}\left(1-\tilde{r}^{2}\right), \\
p_{1}=0 .
\end{gathered}
$$

In particular, in the planar case $\phi_{0}=0, \eta\left(\phi_{0}\right)=g\left(\phi_{0}\right)=\eta_{1}$ and $m\left(\phi_{0}\right)$ $=\gamma_{1}+\gamma_{2}$, while in the homeotropic case $\phi_{0}=\pi / 2, \eta\left(\phi_{0}\right)=g\left(\phi_{0}\right)$ $=\eta_{2}$ and $m\left(\phi_{0}\right)=\gamma_{1}-\gamma_{2}$. In both cases, the first-order radial and vertical velocities, as well as the first-order pressure, are all identically zero since $\Pi_{3}=0$. Analytical expressions for the higher-order terms in these cases can also be readily obtained but are omitted here for brevity.

\section{B. Shear stress and couple stress on the top plate and the substrate}

As mentioned in Sec. I, liquid crystal device performance can be affected by the misalignment of the molecules at the plates. ${ }^{6-8}$
The source of this misalignment is a current topic of research, but there is some evidence that this is an effect of the flow of the liquid crystal during the ODF method. ${ }^{8}$ Flow of a nematic may affect the alignment layer at one or both of the plates through, for example, a frictional force derived from the shear stress at the plates or a director torque derived from the couple stress at the plates. These stresses can be calculated from the director angle and velocity.

The leading-order shear stress, $g\left(\phi_{0}\right) \partial u_{0} / \partial \tilde{z}$, can be obtained from Eqs. (53) - (56) and (59), yielding

$$
\begin{aligned}
& \left.g\left(\phi_{0}\right) \frac{\partial u_{0}}{\partial \tilde{z}}\right|_{\tilde{z}=1}=6 \sqrt{\frac{V}{\pi}} \frac{\eta\left(\phi_{0}\right) h^{\prime}(t)}{h(t)^{3 / 2}} \tilde{r}\left[1-\frac{\int_{0}^{1} \frac{\xi}{g\left(\phi_{0}\right)} \mathrm{d} \xi}{\int_{0}^{1} \frac{1}{g\left(\phi_{0}\right)} \mathrm{d} \xi}\right], \\
& \left.g\left(\phi_{0}\right) \frac{\partial u_{0}}{\partial \tilde{z}}\right|_{\tilde{z}=0}=-6 \sqrt{\frac{V}{\pi}} \frac{\eta\left(\phi_{0}\right) h^{\prime}(t)}{h(t)^{3 / 2}} \tilde{r} \frac{\int_{0}^{1} \frac{\xi}{g\left(\phi_{0}\right)} \mathrm{d} \xi}{\int_{0}^{1} \frac{1}{g\left(\phi_{0}\right)} \mathrm{d} \xi}
\end{aligned}
$$

at the top plate and the substrate, respectively.

The torque on the director depends on the couple stress. From Eqs. (53)-(56), the leading-order couple stress, $\partial \phi_{0} / \partial \tilde{z}$, is constant and is equal to zero for the planar and homeotropic anchoring cases, $\pi / 2$ for the HAN anchoring case, and $\pi$ for the $\pi$-cell anchoring case. The first-order couple stress, $\operatorname{Er} \partial \phi_{1} / \partial \tilde{z}$, can be obtained from Eqs. (53)-(56) and (61), yielding

$$
\begin{aligned}
\left.\frac{\partial \phi_{1}}{\partial \tilde{z}}\right|_{\tilde{z}=1}= & 6 \sqrt{\frac{V}{\pi}} \frac{\eta\left(\phi_{0}\right) h^{\prime}(t)}{h(t)^{1 / 2}} \tilde{r}\left[\int_{0}^{1} m\left(\phi_{0}\right) \frac{\mathrm{d} \Pi_{1}(\xi)}{\mathrm{d} \xi} \mathrm{d} \xi\right. \\
& \left.-\int_{0}^{1} \int_{0}^{\xi} m\left(\phi_{0}\right) \frac{\mathrm{d} \Pi_{1}(\zeta)}{\mathrm{d} \zeta} \mathrm{d} \zeta \mathrm{d} \xi\right], \\
\left.\frac{\partial \phi_{1}}{\partial \tilde{z}}\right|_{\tilde{z}=0}= & -6 \sqrt{\frac{V}{\pi}} \frac{\eta\left(\phi_{0}\right) h^{\prime}(t)}{h(t)^{1 / 2}} \tilde{r} \int_{0}^{1} \int_{0}^{\xi} m\left(\phi_{0}\right) \frac{\mathrm{d} \Pi_{1}(\zeta)}{\mathrm{d} \zeta} \mathrm{d} \zeta \mathrm{d} \xi,
\end{aligned}
$$

at the top plate and the substrate, respectively. The leading-order shear stress and the leading- and first-order couple stresses, for the four anchoring cases, will be described below.

\section{Forces on the top plate and the substrate}

In an experimental or industrial setting, two measurable quantities are the forces on the top plate and on the substrate. Indeed, measuring the force on the top plate is one method used industrially to monitor the distance between plates and hence to determine when the squeezing of the nematic should be stopped. The forces on the top plate and on the substrate can be calculated by integrating the stress tensor over the appropriate boundary. The dimensional forces on the top plate and substrate are defined by

$$
[F]_{z=h(t)}=-\int_{S}\left[\hat{\mathbf{e}}_{z} \cdot \underline{\underline{t}} \cdot \hat{\mathbf{e}}_{z}\right]_{z=h(t)} \mathrm{d} S
$$

and

$$
[F]_{z=0}=-\int_{S}\left[\hat{\mathbf{e}} z \cdot \underline{\mathbf{t}} \cdot \hat{\mathbf{e}}_{z}\right]_{z=0} \mathrm{~d} S
$$


respectively, where the stress tensor $\underline{\underline{t}}$ is expressed in component form as

$$
\begin{aligned}
t_{i j}= & -p \delta_{i j}-\frac{\partial \omega_{F}}{\partial[\nabla \mathbf{n}]_{k j}}[\nabla \mathbf{n}]_{k j}+\alpha_{1} n_{k} e_{k p} n_{p} n_{i} n_{j}+\alpha_{2} N_{i} n_{j} \\
& +\alpha_{3} n_{i} N_{j}+\alpha_{4} e_{i j}+\alpha_{5} n_{j} e_{i k} n_{k}+\alpha_{6} n_{i} e_{j k} n_{k} .
\end{aligned}
$$

In the present asymptotic limit of small Ericksen number, the leading-order term in the stress tensor is simply that due to the pressure, and the nondimensional leading-order force $F_{0}$ evaluated on the top plate and on the substrate is then

$$
\left[F_{0}\right]_{z=h(t)}=\int_{S} p_{0} \mathrm{~d} S \text { and }\left[F_{0}\right]_{z=0}=\int_{S} p_{0} \mathrm{~d} S,
$$

respectively. Using the rescaling equation (21) and substituting the leading-order pressure [Eq. (68)] into Eq. (90) and integrating over the entire top plate or substrate with respect to $\theta$ and $\tilde{r}$ yield

$$
\left[F_{0}\right]_{\tilde{z}=1}=-\left[F_{0}\right]_{\tilde{z}=0}=A p_{\mathrm{I}}-\frac{3 \eta\left(\phi_{0}\right) V^{2} h^{\prime}(t)}{2 \pi h(t)^{5}} .
$$

The leading-order force on the top plate $\left[F_{0}\right]_{\tilde{z}=1}$ is equal and opposite to the leading-order force on the substrate $\left[F_{0}\right]_{\tilde{z}=0}$. Since the effective viscosity $\eta\left(\phi_{0}\right)$ appearing in Eq. (91) does not depend on time, the forces on the top plate and on the substrate increase like $h(t)^{-5}$. As $\eta\left(\phi_{0}\right)$ is largest for the homeotropic anchoring case (see Table III), we find that this anchoring case is associated with the largest forces on the top plate and on the substrate, while the planar anchoring case is associated with the smallest forces on the top plate and on the substrate. In general, Eq. (91) shows that as the film is squeezed and the height of the top plate $h(t)$ reduces, an increasing force is required to move the top plate, and the difference in force required to move the top plate for each anchoring case depends on the value of the effective viscosity $\eta\left(\phi_{0}\right)$ for each anchoring case.

The director, velocity, and pressure, as well as the shear stresses, the couple stresses, and the forces on the top plate and on the substrate, described above all depend on the manner in which the height of the top plate $h(t)$ varies in time. As described previously, there are two important scenarios for the time-dependence of $h(t)$ : the prescribed speed scenario and the prescribed force scenario. The ODF method, in which the motion of the top plate is controlled by a machine which squeezes the film of nematic until there is a prescribed gap between the plates, corresponds to the first scenario. Typically, ODF processes use a constant plate speed, and so this special case is considered in Sec. IV (although the analysis can be readily generalized to other cases). The widely studied problem in which the top plate moves downwards under a constant force due to its own weight is a particular case of the second scenario, and so this special case is considered in Sec. V (although, again, the analysis can be readily generalized to other cases).

\section{RESULTS FOR A PRESCRIBED SPEED}

In the scenario of the top plate moving with a prescribed constant speed, we set the dimensional height of the top plate to be $h(t)=\mathcal{H}-w_{p} t$. The initial dimensional height of the top plate, $\mathcal{H}$, and the constant dimensional top plate speed, $w_{p}$ downwards, can then be used to set the values used in the nondimensionalization given by Eq. (13) in Sec. II B using $\mathcal{U}=w_{p} \sqrt{V /\left(\pi \mathcal{H}^{3}\right)}$. The nondimensionalized height of the top plate is then simply

$$
h(t)=1-t .
$$

For the numerical solutions described in this section, we will set $p_{\mathrm{I}}=0$ and use the material parameter values for the nematic $5 \mathrm{CB}^{12}$ unless stated otherwise.

\section{A. Leading-order radial velocity}

The leading-order radial velocity $u_{0}$, given by Eq. (59), is plotted in Fig. 3 as a function of the original unscaled vertical coordinate $z$ for the four anchoring cases at $r=R(t) / 2$ and $t=0.5$. As Fig. 3 shows, the radial velocity is identical in the planar (solid line) and homeotropic (dotted line) anchoring cases, with both of these anchoring cases having a symmetric Poiseuille flow. As Fig. 3 also shows, the leading-order radial velocity for the HAN anchoring case (dashed line) has a Poiseuille-like profile, with the flow skewed toward the lower viscosity region in the lower part of the squeeze film. For the $\pi$-cell anchoring case (dashed-dotted line), Fig. 3 shows similar behavior, with lower velocity in the higher viscosity region in the center of the squeeze film and a higher velocity in the lower viscosity regions near the substrate and the top plate. The location of the maximum radial velocity, denoted by $z=z^{*}$, can be found using Eq. (74) and the rescaling equation (21) to be

$$
z^{*}=h(t) \frac{\int_{0}^{1} \frac{\xi}{g\left(\phi_{0}\right)} \mathrm{d} \xi}{\int_{0}^{1} \frac{1}{g\left(\phi_{0}\right)} \mathrm{d} \xi}
$$

Clearly, from Eq. (93), the location of the maximum velocity is a constant fraction $h(t)$ and so varies with time $t$ like $h(t)$. In the planar, homeotropic, and $\pi$-cell (but not the HAN) anchoring cases, it is straight forward to show that $z^{*}=h(t) / 2$ (i.e., the maximum velocity is always in the center of the squeeze film).

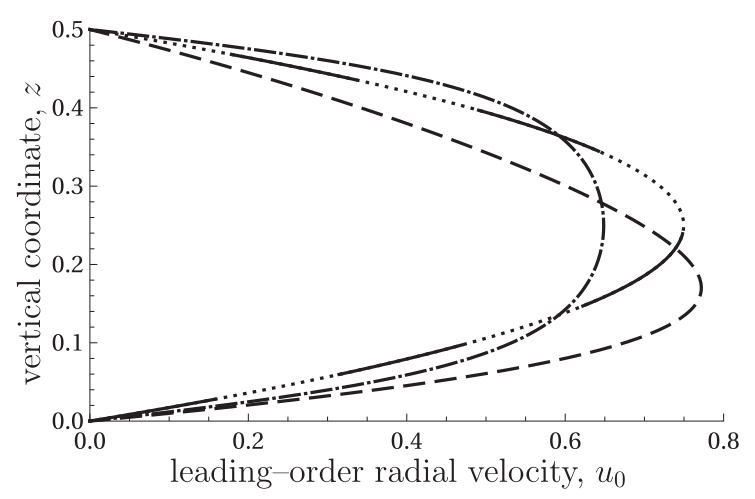

FIG. 3. Leading-order radial velocity $u_{0}$, given by Eq. (59), plotted as a function of the vertical coordinate $z$ for the four anchoring cases: planar (solid), homeotropic (dotted), HAN (dashed), and $\pi$-cell (dashed-dotted) at $r=R(t) / 2$ and $t=0.5$ using $p_{1}=0$ and the material parameter values for the nematic $5 \mathrm{CB} .{ }^{12}$ The results for the planar and homeotropic anchoring cases are identical, indicated by the solid and dotted curves being plotted intermittently. 
For the HAN and $\pi$-cell anchoring cases, the higher velocity in the lower viscosity regions leads to changes in the shear stress at the substrate and at the top plate, as indicated by the gradient $\partial u_{0} / \partial z$ at $z=0$ and $z=h(t)$ in Fig. 3, when compared to the Poiseuille flow in the planar and homeotropic anchoring cases.

The evolution of the leading-order radial velocity $u_{0}$, given by Eq. (59), is plotted in Fig. 4 as a function of $z$ at $r=R(t) / 2$ for $t=0, t=0.2, t=0.4$, and $t=0.6$ for each of the four anchoring cases. For each of the four anchoring cases, the radial velocity retains the same functional form shown in Fig. 3 but increases in magnitude as time increases. As Eq. (76) shows, this increase in magnitude is proportional to $h^{\prime}(t) / h(t)^{3 / 2}$.

\section{B. Leading-order vertical velocity}

The leading-order vertical velocity $w_{0}$, given by Eq. (65), is plotted in Fig. 5 as a function of $z$ for the four anchoring cases at $r=R(t) / 2$ and $t=0.5$. The behavior of the leading-order vertical flow can be understood by considering the leading-order radial velocity shown in Fig. 3. By conservation of mass, a reduction in the radial velocity must be matched by an increase in the vertical velocity. The leading-order radial velocity is identical for the planar and homeotropic anchoring cases; this leads to the leading-order vertical velocity also being identical, as shown in Eq. (80). As Fig. 5 shows, for the HAN anchoring case, the leading-order vertical velocity is larger in magnitude at all values of $z$ than any of the other three anchoring cases. This is due to the leading-order radial velocity being skewed toward the lower part of the squeeze film, leading to a larger vertical flux into this lower viscosity region and thus a larger downward vertical velocity. In the $\pi$-cell anchoring case, there is a smaller vertical velocity in the upper half $[h(t) / 2<z<h(t)]$ of the squeeze film and a larger velocity in the lower half $[0<z<h(t) / 2]$ than in the planar and homeotropic anchoring cases. As shown in Fig. 3, there is larger radial velocity near the top plate in the $\pi$-cell anchoring case than in the planar and homeotropic anchoring cases resulting in smaller vertical flow in the $\pi$-cell anchoring case than in the planar and homeotropic anchoring cases. The vertical velocity near the substrate is larger in the $\pi$-cell anchoring case than in the planar and homeotropic anchoring cases, as the radial velocity in the middle of the squeeze film is smaller, as shown in Fig. 3, in the $\pi$-cell anchoring case than that of the planar and homeotropic anchoring cases. Plots of the evolution of the leading-order vertical

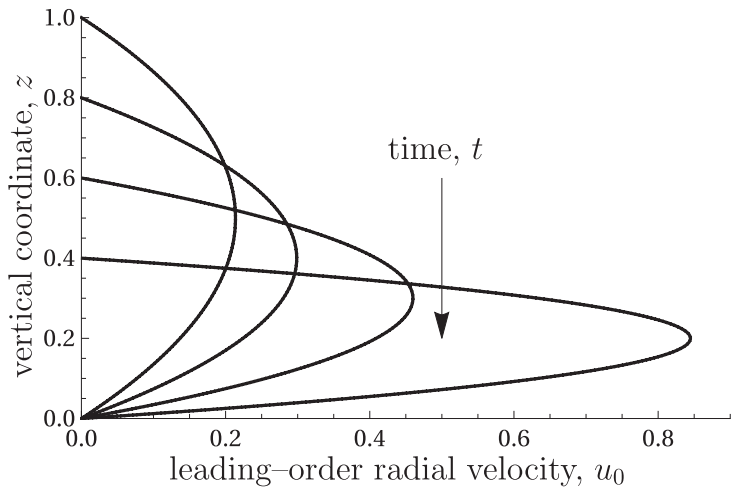

(a) planar anchoring case

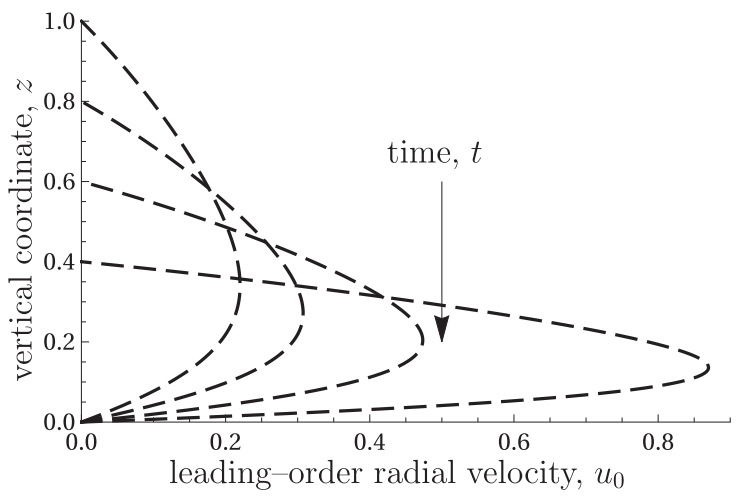

(c) HAN anchoring case

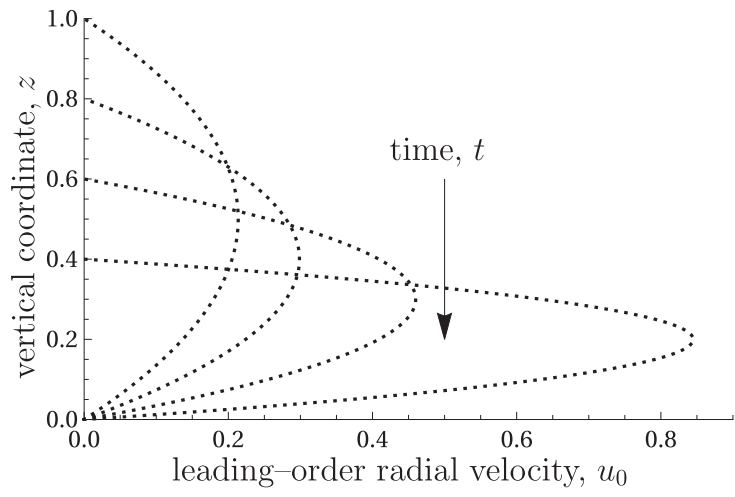

(b) homeotropic anchoring case

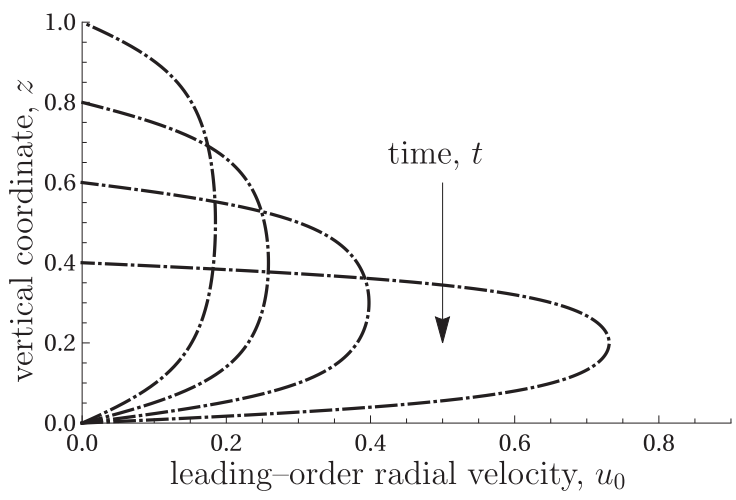

(d) $\pi$-cell anchoring case

FIG. 4. The evolution of the leading-order radial velocity $u_{0}$, given by Eq. (59), plotted as a function of vertical coordinate $z$ for the four anchoring cases: (a) planar (solid), (b) homeotropic (dotted), (c) HAN (dashed), and (d) $\pi$-cell (dashed-dotted) at $r=R(t) / 2$ for $t=0, t=0.2, t=0.4$, and $t=0.6$ using $p_{1}=0$ and the material parameter values for the nematic $5 \mathrm{CB}$. 


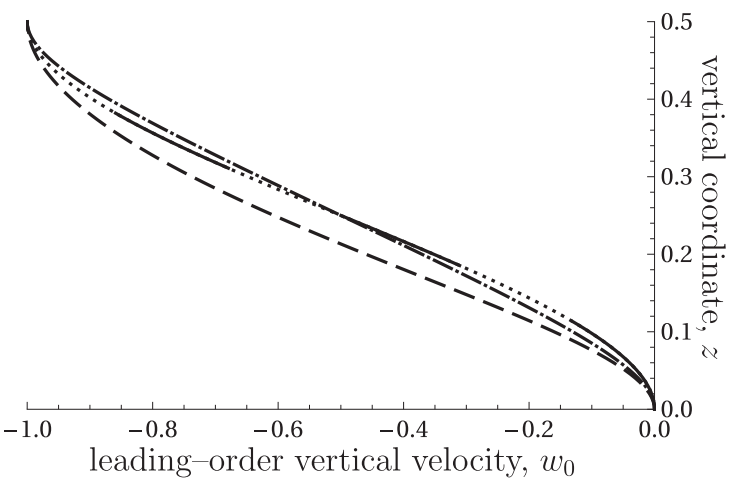

FIG. 5. Leading-order vertical velocity $w_{0}$, given by Eq. (65), plotted as a function of vertical coordinate $z$ for the four anchoring cases: planar (solid), homeotropic (dotted), HAN (dashed), and $\pi$-cell (dashed-dotted) at $r=R(t) / 2$ and $t=0.5$ using $p_{l}=0$ and the material parameter values for the nematic $5 \mathrm{CB} .{ }^{12}$ The results for the planar and homeotropic anchoring cases are identical, indicated by the solid and dotted curves being plotted intermittently.

velocity $w_{0}$, given by Eq. (65), are omitted since, as Eqs. (76) and (92) show, the leading-order vertical velocity does not depend on time in the prescribed speed scenario.

\section{Leading-order pressure}

The leading-order pressure $p_{0}$, given by Eq. (68), is plotted in Fig. 6 as a function of $r$ for the four anchoring cases at $t=0.5$. The results given in Table III show that the homeotropic anchoring case has the largest effective viscosity and hence the highest pressure, whilet the planar anchoring case has the smallest effective viscosity and hence the lowest pressure, in agreement with Fig. 6. The pressures in the HAN and the $\pi$-cell anchoring cases lie between those in the planar and homeotropic anchoring cases, with the pressure in the HAN case being larger due to it having a larger effective viscosity.

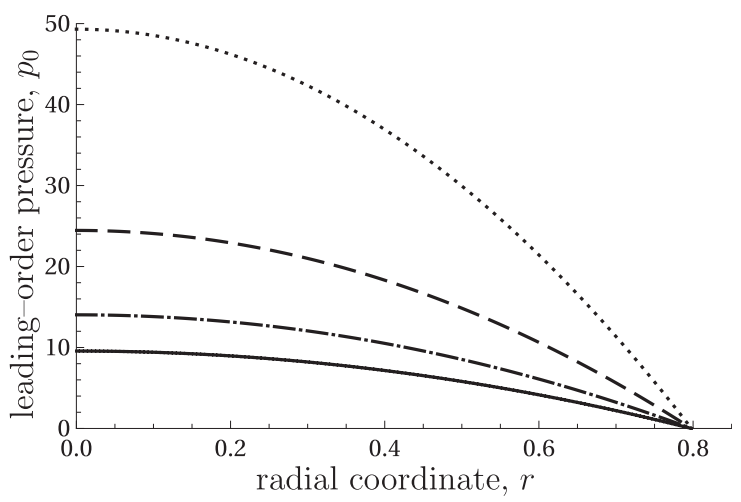

FIG. 6. Leading-order pressure $p_{0}$, given by Eq. (68), plotted as a function of radial coordinate $r$ for the four anchoring cases: planar (solid), homeotropic (dotted), HAN (dashed), and $\pi$-cell (dashed-dotted) at $t=0.5$ using $p_{1}=0$ and the material parameter values for the nematic $5 \mathrm{CB}$.

\section{First-order director angle}

As we have seen, since the Ericksen number is small, the director angle is dominated by elastic effects and flow has no effect on the leading-order director angle. However, at higher orders, the flow has an effect on the director angle. The first-order director angle $\phi_{1}$, given by Eq. (61), is plotted in Fig. 7 as a function of $z$ for the four anchoring cases at $r=R(t) / 2$ and $t=0.5$. As Fig. 7 shows, the planar and homeotropic anchoring cases exhibit a flow-aligning correction to the leading-order director angle in response to the leading-order radial velocity shown in Fig. 3. For the planar anchoring case (solid line), as a consequence of the leading-order Poiseuille flow profile, the leading-order solution $\phi_{0}=0$ is increased toward the positive Leslie angle $\phi_{\mathrm{L}}$ in the lower half $[0<z<h(t) / 2]$ of the squeeze film and decreased toward the negative Leslie angle $-\phi_{\mathrm{L}}$ in the upper half $[h(t) / 2<z<h(t)]$ of the squeeze film. The homeotropic anchoring case (dotted line) has a similar behavior, the leading-order solution $\phi_{0}=\pi / 2$ is decreased toward the positive Leslie angle $\phi_{\mathrm{L}}$ in the lower half of the squeeze film and increased towards the closest negative Leslie angle $\pi-\phi_{\mathrm{L}}$ in the upper half of the squeeze film. (Recall that the terms "positive" and "negative" Leslie angles refer to the sign of the shear rate that is flow aligning the director rather than to the sign of the numerical value of the angle.) The perturbation in the homeotropic anchoring case is larger than that in the planar anchoring case since the closest Leslie angle for the nematic 5CB is $\phi_{\mathrm{L}} \approx 0.208$, which is closer to 0 than to $\pi / 2$, so that the torque applied to the director, and hence the perturbation to the director angle, due to the flow is larger in the homeotropic anchoring case.

The behavior in the HAN anchoring case (dashed line) is more complicated. Close to the top plate, where the leading-order director is similar to the leading-order director for the homeotropic anchoring case, the behavior of the first-order director angle is similar to that in the homeotropic case (denoted by the dotted line in Fig. 7), such that, in this region, the flow acts to align the director toward the nearest negative Leslie angle $\pi-\phi_{\mathrm{L}} \approx 2.933$, resulting in a positive perturbation $\phi_{1}$. Indeed, because the shear rate is negative over the majority of the squeeze film (see Fig. 3), the torque in the majority of the cell will be positive, tending to increase the first-order director angle. However, close to the substrate at $z=0$, the torque applied

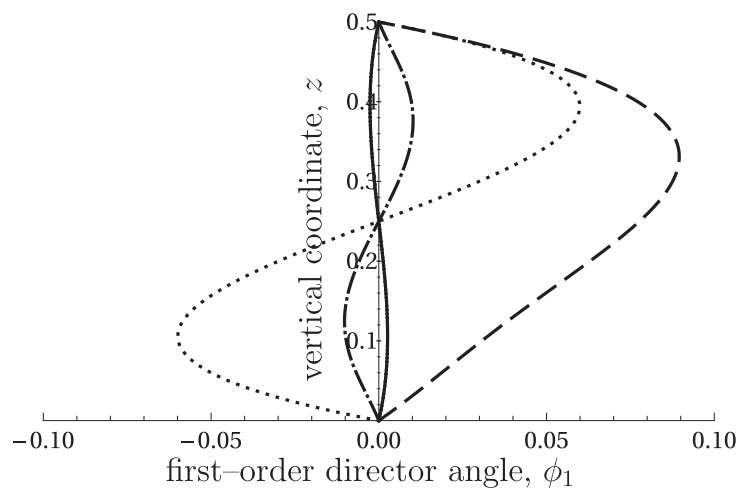

FIG. 7. First-order director angle $\phi_{1}$, given by Eq. (61), plotted as a function of vertical coordinate $z$ for the four anchoring cases: planar (solid), homeotropic (dotted), HAN (dashed), and $\pi$-cell (dashed-dotted) at $r=R(t) / 2$ and $t=0.5$ using $p_{1}=0$ and the material parameter values for the nematic $5 \mathrm{CB}$. 
to the director will attempt to align the director toward the positive Leslie angle $\phi_{\mathrm{L}} \approx 0.208$. Therefore, for leading-order director angles less than $\phi_{\mathrm{L}}$, this will lead to a positive first-order perturbation $\phi_{1}$, whereas for leading-order director angles greater than $\phi_{\mathrm{L}}$, this should lead to a negative first-order perturbation $\phi_{1}$. However, the strength of the elasticity and the large positive torque in the rest of the squeeze film means that the net result is a positive perturbation $\phi_{1}$ throughout the squeeze film.

For the $\pi$-cell anchoring case, in the upper half of the squeeze film the leading-order director angle is greater than $\pi / 2$ and in the lower half of the squeeze film the leading-order director angle is less than $\pi / 2$. Therefore, in the upper half of the squeeze film, the torque applied to the director will lead to a positive perturbation $\phi_{1}$ toward the nearest negative Leslie angle $\pi-\phi_{\mathrm{L}}$, and in the lower half of the squeeze film the torque applied to the director will lead to a negative perturbation $\phi_{1}$ toward the nearest positive Leslie angle $\phi_{\mathrm{L}}$.

The evolution of the first-order director angle $\phi_{1}$, given by Eq. (61), is plotted in Fig. 8 as a function of $z$ for the four anchoring cases at $r=R(t) / 2$ for $t=0, t=0.2, t=0.4$, and $t=0.6$. As the squeezing occurs, the shear rate increases, leading to an increase in the torque on the director, and thus, the magnitude of the first-order director angle $\left|\phi_{1}\right|$ increases as the shear gradient aligns the director closer toward the closest Leslie angle. As Eq. (76) shows, this increase in magnitude of $\phi_{1}$ is proportional to $h^{\prime}(t) / h(t)^{1 / 2}$.

In order to visualize the perturbations of the leading-order director due to flow, Fig. 9 shows the leading-order director field $\mathbf{n}\left(\phi_{0}\right)$ and the director field up to first-order $\mathbf{n}\left(\phi_{0}+\operatorname{Er} \phi_{1}\right)$. Note that in order to clearly show the first-order perturbation to the leadingorder director field, we have exaggerated the first-order perturbation by artificially increasing the Ericksen number to $\mathrm{Er}=100$ in Fig. 9(b), Er $=10$ in Figs. 9(d) and 9(f), and Er $=35$ in Fig. 9(h).

\section{E. Shear stress and couple stress on the top plate and the substrate}

The leading-order shear stress $g\left(\phi_{0}\right) \partial u_{0} / \partial \tilde{z}$ at the top plate $\tilde{z}=1$ and the substrate $\tilde{z}=0$, given by Eqs. (84) and (85), and the leading- and first-order couple stress $\partial \phi_{0} / \partial \tilde{z}+\operatorname{Er} \partial \phi_{1} / \partial \tilde{z}$ at $\tilde{z}=1$ and $\tilde{z}=0$, given by Eqs. (86) and (87), are shown in Table IV for the four anchoring cases, at $r=R(t) / 2$ and $t=0.5$, and using material parameter values for the nematic $5 \mathrm{CB} .{ }^{12}$ Table IV shows that

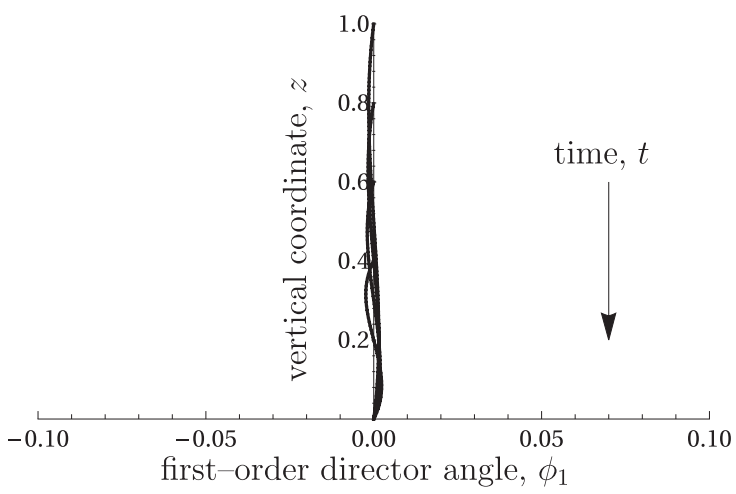

(a) planar anchoring case

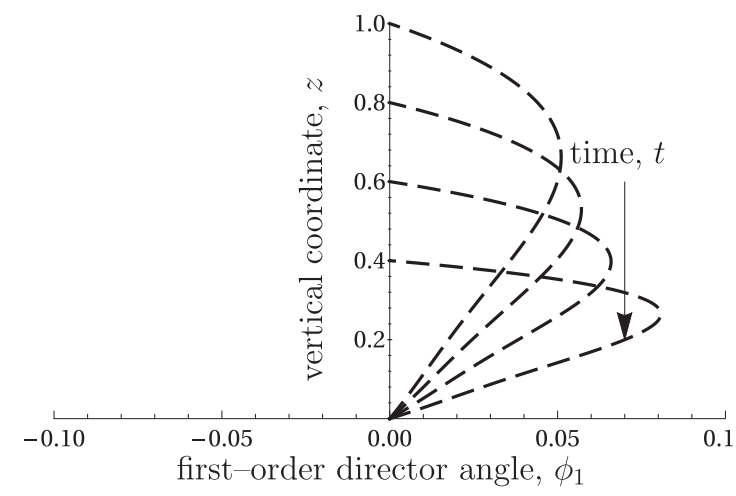

(c) HAN anchoring case

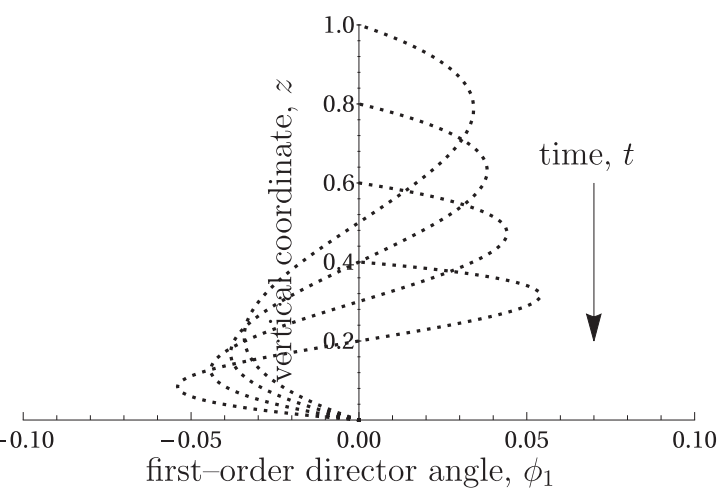

(b) homeotropic anchoring case

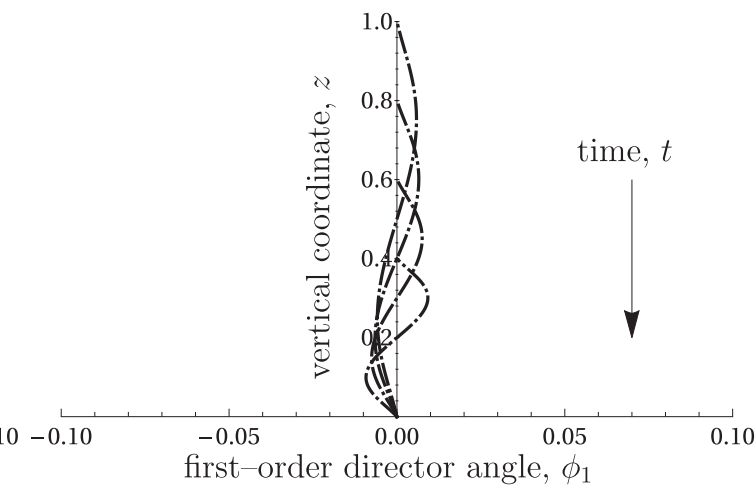

(d) $\pi$-cell anchoring case

FIG. 8. The evolution of the first-order director angle $\phi_{1}$, given by Eq. (61), plotted as a function of vertical coordinate $z$ for the four anchoring cases: (a) planar (solid), (b) homeotropic (dotted), (c) HAN (dashed), and (d) $\pi$-cell (dashed-dotted) at $r=R(t) / 2$ for $t=0, t=0.2, t=0.4$, and $t=0.6$ using $p_{1}=0$ and the material parameter values for the nematic $5 \mathrm{CB} .{ }^{12}$ The arrow shows the direction of increasing time, $t$. 


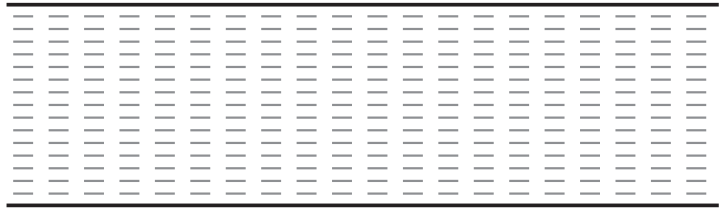

(a) planar anchoring $\mathbf{n}\left(\phi_{0}\right)$

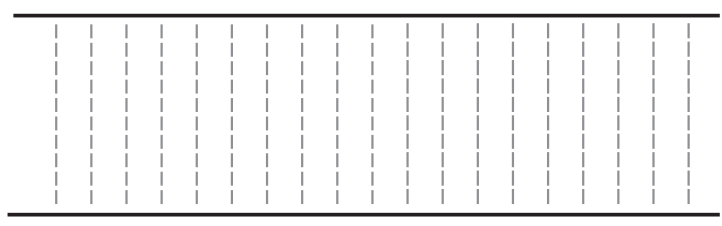

(c) homeotropic anchoring $\mathbf{n}\left(\phi_{0}\right)$

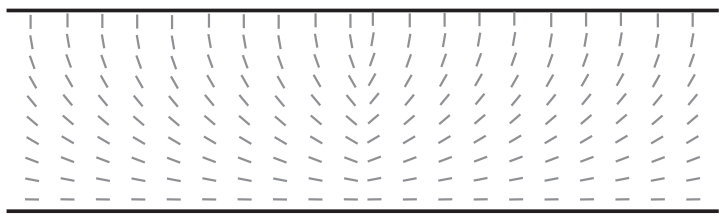

(e) HAN anchoring $\mathbf{n}\left(\phi_{0}\right)$

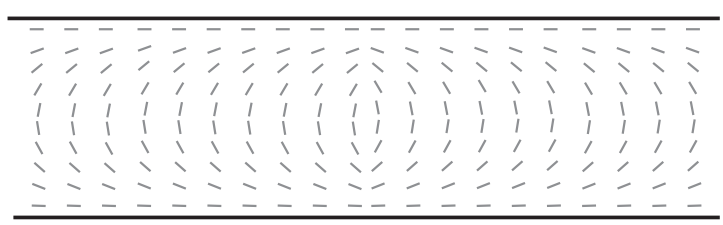

(g) $\pi$-cell anchoring $\mathbf{n}\left(\phi_{0}\right)$

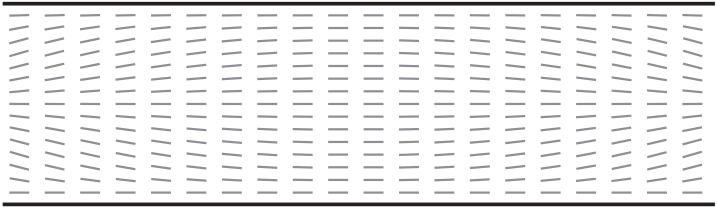

(b) planar anchoring $\mathbf{n}\left(\phi_{0}+\operatorname{Er} \phi_{1}\right)$

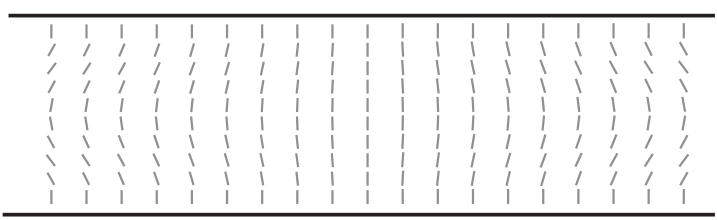

(d) homeotropic anchoring $\mathbf{n}\left(\phi_{0}+\operatorname{Er} \phi_{1}\right)$

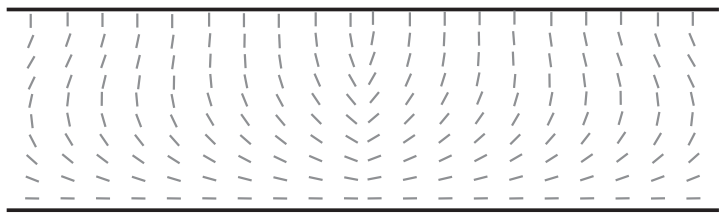

(f) HAN anchoring $\mathbf{n}\left(\phi_{0}+\operatorname{Er} \phi_{1}\right)$

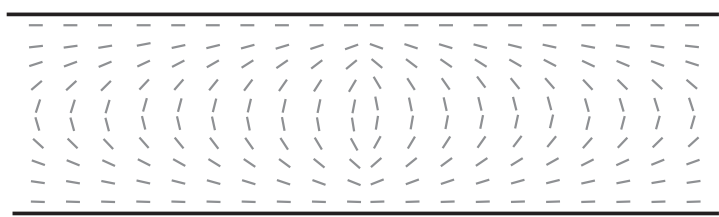

(h) $\pi$-cell anchoring $\mathbf{n}\left(\phi_{0}+\operatorname{Er} \phi_{1}\right)$

FIG. 9. The leading-order director field $\mathbf{n}\left(\phi_{0}\right)[(\mathrm{a}),(\mathrm{c}),(\mathrm{e})$, and $(\mathrm{g})]$ and the director field up to first-order $\mathbf{n}\left(\phi_{0}+\operatorname{Er} \phi_{1}\right)[(\mathrm{b})$, (d), (f), and (h)] for the four anchoring cases: [(a) and (b)] planar, [(c) and (d)] homeotropic, $\left[(\mathrm{e})\right.$ and (f)] HAN, and [(g) and (h)] $\pi$-cell at $t=0.09$, where $\mathrm{Er}=100 \mathrm{in}(\mathrm{b}), \mathrm{Er}=10$ in (d) and (f), and $\mathrm{Er}=35$ in (h) using $p_{\mathrm{l}}=0$ and the material parameter values for the nematic $5 \mathrm{CB} .{ }^{12}$ Note that in order to clearly show the first-order perturbation to the leading-order director field, we have exaggerated the first-order perturbation by artificially increasing the Ericksen number.

TABLE IV. Leading-order shear stress $g\left(\phi_{0}\right) \partial u_{0} / \partial \tilde{z}$ at the top plate $\tilde{z}=1$ and the substrate $\tilde{z}=0$, given by Eqs. (84) and (85), and the leading- and first-order couple stress $\partial \phi_{0} / \partial \tilde{z}+\operatorname{Er} \partial \phi_{1} / \partial \tilde{z}$ at $\tilde{z}=1$ and $\tilde{z}=0$, given by Eqs. (86) and (87), evaluated for the four anchoring cases: planar, homeotropic, HAN, and $\pi$-cell, at $r=R(t) / 2$ and $t=0.5$, using the material parameter values for the nematic $5 \mathrm{CB} .^{12}$

\begin{tabular}{lccccc}
\hline \hline & \multicolumn{2}{c}{ Shear stress $g\left(\phi_{0}\right) \partial u_{0} / \partial \tilde{z}$} & & \multicolumn{2}{c}{ Couple stress $\partial \phi_{0} / \partial \tilde{z}+\operatorname{Er} \partial \phi_{1} / \partial \tilde{z}$} \\
\cline { 2 - 3 } \cline { 5 - 6 } Anchoring case & $\tilde{z}=1$ & $\tilde{z}=0$ & & $\tilde{z}=1$ & $\tilde{z}=0$ \\
\hline Planar & -1.1952 & 1.1952 & & $0.0173 \mathrm{Er}$ & $0.0173 \mathrm{Er}$ \\
Homeotropic & -6.1637 & 6.1637 & & $-0.3967 \mathrm{Er}$ & $-0.3967 \mathrm{Er}$ \\
HAN & -4.0358 & 2.0786 & & $\pi / 2-0.3855 \mathrm{Er}$ & $\pi / 2+0.1076 \mathrm{Er}$ \\
$\pi$-cell & -1.7543 & 1.7543 & & $\pi-0.0315 \mathrm{Er}$ & $\pi-0.0315 \mathrm{Er}$ \\
\hline \hline
\end{tabular}




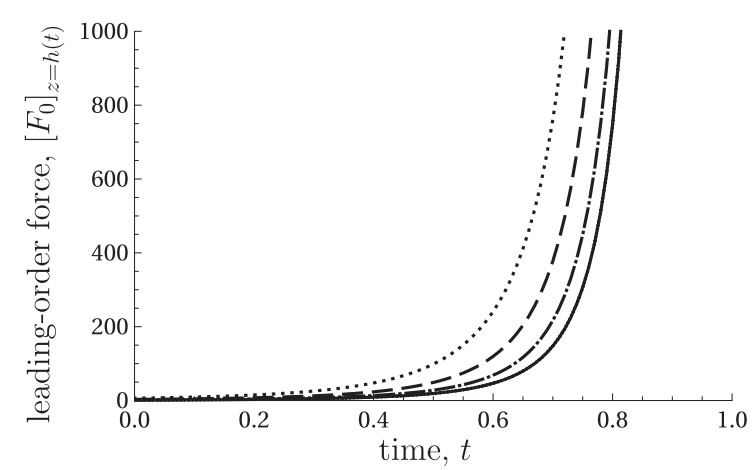

FIG. 10. Leading-order force on the top plate $\left[F_{0}\right]_{z=h(t)}$, given by Eq. (91), plotted as a function of time $t$ for the four anchoring cases: planar (solid), homeotropic (dotted), HAN (dashed) and $\pi$-cell (dashed-dotted) using $p_{1}=0$ and the material parameter values for the nematic $5 \mathrm{CB}$.

the leading-order shear stress is largest for the homeotropic anchoring case, which is due to the large value of $g\left(\phi_{0}\right)$ at both $\tilde{z}=1$ and $\tilde{z}=0$ in this case. The HAN anchoring case is the only case with an asymmetric solution for $u_{0}$, as shown in Fig. 3 , which gives rise to a corresponding asymmetry in the shear stress at $\tilde{z}=1$ and $\tilde{z}=0$. For the planar, homeotropic, and $\pi$-cell anchoring cases, the solution for $\phi_{1}$ is antisymmetric about $\tilde{z}=0.5$, as shown in Fig. 7 , which leads to equal first-order couple stresses at $\tilde{z}=1$ and $\tilde{z}=0$ in these cases. For the HAN anchoring case, the solution for $\phi_{1}$ is asymmetric, leading to different values of the couple stress at $\tilde{z}=1$ and $\tilde{z}=0$. As Eqs. (84)-(87) show, the magnitude of the leadingorder shear stress and the first-order couple stress increase as $h^{\prime}(t) / h(t)^{3 / 2} \tilde{r}$ and $h^{\prime}(t) / h(t)^{1 / 2} \tilde{r}$, respectively.

\section{F. Leading-order force on the top plate and the substrate}

The leading-order force on the top plate $\left[F_{0}\right]_{z=h(t)}$, given by Eq. (91), is plotted in Fig. 10 as a function of time for the four anchoring cases. This is the force required to squeeze the nematic film at a constant prescribed speed. The leading-order force on the substrate is equal and opposite to the leading-order force on the top plate, i.e., $\left[F_{0}\right]_{z=0}=-\left[F_{0}\right]_{z=h(t)}$. As Fig. 10 shows, an increasing magnitude of force must be applied to the top plate to close the squeeze film at a constant prescribed speed. Indeed, as is immediately evident from Eq. (91), the force needed to maintain a constant prescribed speed increases as $h(t)^{-5}=(1-t)^{-5}$ for all anchoring cases and so approaches infinity as $t$ approaches the time $t=1$ at which the top plate meets the substrate. It is also evident from Fig. 10 that the anchoring case that produces the largest pressure requires the largest force to close the squeeze film, so that the homeotropic anchoring case requires the largest force to close the squeeze film to a given height of the top plate, and therefore requires the most work during manufacturing.

\section{RESULTS FOR A PRESCRIBED FORCE}

The second scenario studied is that in which the top plate is free to move under a prescribed constant force due to its own dimensional weight. Although this is not the situation in the ODF method, it is the more commonly studied situation for a Newtonian fluid and is of scientific interest in its own right. The initial dimensional height of the top plate, $\mathcal{H}$, and the constant dimensional weight of the top plate, $W_{p}$, can then be used to set the values used in the nondimensionalization given by Eq. (13) in Sec. II B using $\mathcal{U}=W_{p} \mathcal{H}^{2} / \mu \mathcal{R}^{3}$. The nondimensionalized initial height of the top plate is then $h=1$ at $t=0$. Unlike the previous scenario, for $t>0$, the height of the top plate $h(t)$ is now unknown and must be determined by considering the balance of forces on the top plate. In the limit of small Ericksen number, we seek a solution for the height of the top plate $h(t)$ as an asymptotic expansion in the form

$$
h(t)=h_{0}(t)+\mathcal{O}(\mathrm{Er})
$$

The force on the top plate, given by Eq. (91), can then be used to calculate the unknown leading-order height of the top plate $h_{0}(t)$ by equating the sum of the weight of the top plate and the force from a fixed constant external ambient pressure, $p_{\mathrm{E}}$, with the leadingorder force, namely, $\left[F_{0}\right]_{z=h(t)}=W_{p}+A p_{\mathrm{E}}$. Substituting the asymptotic expansion for the height of the top plate Eq. (94) into Eq. (91) at leading order in Er, we obtain

$$
W_{p}+A p_{\mathrm{E}}=A p_{\mathrm{I}}-\frac{3 \eta\left(\phi_{0}\right) V^{2}}{2 \pi h_{0}^{5}} \frac{\mathrm{d} h_{0}}{\mathrm{~d} t} .
$$

Rearranging, setting $\tilde{W}_{p}=W_{p}-A\left(p_{I}-p_{E}\right)$, and integrating Eq. (95) with respect to $t$, we obtain the solution from leading-order height of the top plate,

$$
h_{0}=\frac{1}{(1+\zeta t)^{1 / 4}}, \quad \text { where } \quad \zeta=\frac{8 \pi \tilde{W}_{p}}{3 \eta\left(\phi_{0}\right) V^{2}}
$$

and $\eta\left(\phi_{0}\right)$ is the effective viscosity defined in Eq. (72). As mentioned previously, typically the ODF method is carried out in a vacuum where $p_{\mathrm{I}}=p_{\mathrm{E}}=0$ so that $\tilde{W}_{p}=W_{p}$ and hence the leading-order height of the top plate decreases in time; however, we note that for $A\left(p_{\mathrm{I}}-p_{\mathrm{E}}\right)>W_{p}$, the leading-order height of the top plate increases in time, and for $A\left(p_{\mathrm{I}}-p_{\mathrm{E}}\right)=W_{p}$, the leading-order height of the top plate remains fixed at $h_{0}=1$. We note that upon substituting $\eta\left(\phi_{0}\right)$ $=1$, the classical solution for height of the top plate for a Newtonian fluid is recovered.

In this work, we have only considered four infinite anchoring cases. However, note that the expression (96) is more general than this and, in fact, represents the leading-order height of the top plate $h_{0}(t)$ for any $\phi_{0}$ that is the solution of Eq. (45) and any anchoring condition (for example, a weak anchoring condition ${ }^{28}$ ). The leadingorder height of the top plate $h_{0}$, given by Eq. (96), is plotted in Fig. 11 as a function of time $t$ for the four anchoring cases using $p_{\mathrm{I}}=p_{\mathrm{E}}$. Figure 11 shows that $h_{0}$ reduces fastest for the planar anchoring case. This result is as might have been expected, since the planar anchoring case has the smallest effective viscosity $\eta\left(\phi_{0}\right)$, and so requires the smallest force to close it at the same rate as the other anchoring cases. The results for each of the anchoring cases differ due to the values of the effective viscosity shown in Table III. As is evident from Eq. (96), the smaller the effective viscosity, the faster the $h_{0}$ decreases.

Using the leading-order height of the top plate $h_{0}$, given by Eq. (96), the solutions for the first-order director angle $\phi_{1}$, the leading-order radial velocity $u_{0}$, the leading-order vertical 


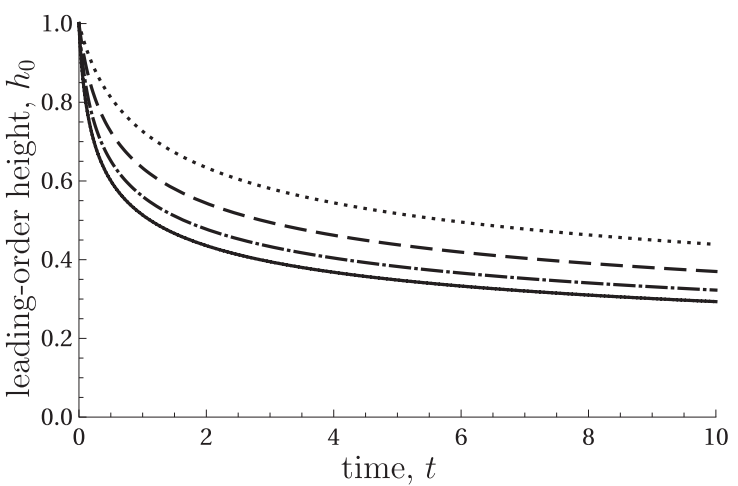

FIG. 11. The leading-order height of the top plate $h_{0}$ in the prescribed force scenario, given by Eq. (96), plotted as a function of time $t$ for the four anchoring cases: planar (solid), homeotropic (dotted), HAN (dashed), and $\pi$-cell (dasheddotted) using $p_{1}=p_{E}, W_{p}=1$, and $V=1$ and the material parameter values for the nematic $5 \mathrm{CB}$.

velocity $w_{0}$, and the leading-order pressure $p_{0}$ in the prescribed force scenario are

$$
\begin{aligned}
\phi_{1}(\tilde{r}, \tilde{z}, t) & =-\frac{3}{2} \zeta \eta\left(\phi_{0}\right) \sqrt{\frac{V}{\pi}} \frac{\Pi_{2}(\tilde{z}) \tilde{r}}{(1+\zeta t)^{9 / 8}}, \\
u_{0}(\tilde{r}, \tilde{z}, t) & =-\frac{3}{2} \zeta \eta\left(\phi_{0}\right) \sqrt{\frac{V}{\pi}} \frac{\Pi_{1}(\tilde{z}) \tilde{r}}{(1+\zeta t)^{7 / 8}} \\
w_{0}(\tilde{z}, t) & =3 \zeta \eta\left(\phi_{0}\right) \sqrt{\frac{V}{\pi}} \frac{\int_{0}^{\tilde{z}} \Pi_{1}(\xi) \mathrm{d} \xi}{(1+\zeta t)^{5 / 4}} \\
p_{0}(\tilde{r}, t) & =p_{\mathrm{I}}+\frac{3 V \zeta \eta\left(\phi_{0}\right)}{4 \pi} \frac{\left(1-\tilde{r}^{2}\right)}{(1+\zeta t)^{1 / 4}}
\end{aligned}
$$

As Eqs. (97)-(100) show, the first-order director angle $\phi_{1}$, the leading-order radial velocity $u_{0}$, the leading-order vertical velocity $w_{0}$, and the leading-order pressure difference $p_{0}-p_{\mathrm{I}}$, all tend toward zero in the limit $t \rightarrow \infty$.

The leading-order shear stress and the leading- and first-order couple stresses at the top plate and the substrate for the four anchoring cases can be calculated using Eqs. (97) and (98). However, since their behavior is qualitatively the same as that in the prescribed speed scenario, the details are omitted for brevity.

\section{SUMMARY AND CONCLUSIONS}

Motivated by the need for a better fundamental understanding of the reorientation of the molecules due to the flow of the liquid crystal during the industrial manufacture of liquid crystal devices, in this work we formulated and analyzed a squeeze-film model for the ODF method. Specifically, we considered a nematic squeeze film in the asymptotic regime in which the drop is thin, inertial effects are weak, and elasticity effects are strong (i.e., in which the aspect ratio $\delta \ll 1$, the reduced Reynolds number $\operatorname{Re} \ll 1$, and the Ericksen number $\mathrm{Er} \ll 1$ are all small) for four specific anchoring cases at the top plate and the substrate (namely, planar, homeotropic, HAN, and $\pi$-cell infinite anchoring conditions) and for two different scenarios for the motion of the top plate (namely, prescribed speed and prescribed force). Analytical expressions for the leading- and firstorder director angles, $\phi_{0}$ and $\phi_{1}$, radial velocity, $u_{0}$ and $u_{1}$, vertical velocity, $w_{0}$ and $w_{1}$, and pressure, $p_{0}$ and $p_{1}$, were obtained and interpreted in terms of the effective viscosity $\eta\left(\phi_{0}\right)$ given by Eq. (72), and the relevant Leslie angles, $\phi_{L},-\phi_{L}$ and $\pi-\phi_{L}$, where $\phi_{L}$ is given by Eq. (52).

The results obtained in this work help us to improve our understanding of the ODF method. Specifically, as mentioned in Sec. I, the misalignment of the molecules at the plates due to the flow of the liquid crystal has been proposed as a possible mechanism for the formation of mura. The present results capture the flow-driven reorientation of the molecules during squeezing via the first-order director angle $\phi_{1}$. In particular, we found that the magnitude of $\phi_{1}$ increases like $h^{\prime}(t) / h(t)^{1 / 2}$ and that the behavior of $\phi_{1}$ depends on the anchoring case. Specifically, the magnitude of $\phi_{1}$ is largest for the homeotropic and HAN anchoring cases, suggesting that these cases are potentially more susceptible to the formation of mura. As also mentioned in Sec. I, damage to the molecular alignment at the plates has also been proposed as a possible mechanism for the formation of mura. If this is the case, the molecular alignment at the plates might be related to the shear stress and/or the couple stress. The leading-order couple stress is zero for the planar and homeotropic anchoring cases, $\pi / 2$ for the HAN anchoring case, and $\pi$ for the $\pi$-cell anchoring case. The leading-order shear stress and the firstorder couple stress on the top plate and the substrate are given by Eqs. (84)-(87). In particular, we found that the magnitudes of the leading-order shear stress and the first-order couple stress increase as $h^{\prime}(t) / h(t)^{3 / 2} \tilde{r}$ and $h^{\prime}(t) / h(t)^{1 / 2} \tilde{r}$, respectively, suggesting that the formation of mura will more likely occur when the top plate speed, $h^{\prime}(t)$, is large; the height of the top plate, $h(t)$, is small; and at a large radius, $\tilde{r}$.

The force required to squeeze the nematic film at a constant prescribed speed is proportional to the effective viscosity, and so in the scenario in which the top plate moves with a constant prescribed speed, the homeotropic anchoring case requires the largest force and the planar condition the smallest force. Correspondingly, in the scenario in which the top plate moves downwards under its own weight, the height of the top plate reduces fastest for the planar anchoring case and slowest for the homeotropic anchoring case. Note that although we only considered four specific anchoring cases of infinite anchoring in this work, the solutions for $\phi_{0}, \phi_{1}, u_{0}, u_{1}, w_{0}$, $p_{0}$, and $p_{1}$ also hold for other anchoring conditions, including weak anchoring.

Finally, it should be pointed out that while in this work we have focused on the possible future manufacturing regime in which elasticity effects are stronger than the viscous ones (i.e., in which the Ericksen number Er $\ll 1$ is small), in Sec. II E we showed that in the current manufacturing regime elasticity effects are typically weaker than the viscous ones (i.e., the Ericksen number Er $\gg 1$ is typically large), and so this asymptotic regime is also of considerable practical interest.

\section{ACKNOWLEDGMENTS}

This work was supported by the United Kingdom Engineering and Physical Sciences Research Council, the University of 
Strathclyde, and Merck KGaA (via EPSRC research Grant No. EP/P51066X/1). The authors gratefully acknowledge the assistance of Dr. David J. Allwright of the Smith Institute for helping to facilitate the present collaboration between the University of Strathclyde and Merck KGaA.

\section{REFERENCES}

${ }^{1}$ X.-D. Mi and D.-K. Yang, "Capillary filling of nematic liquid crystals," Phys. Rev. E 58, 1992-2000 (1998)

${ }^{2}$ H. Kamiya, K. Tajima, K. Toriumi, K. Terada, H. Inoue, T. Yokoue, N. Shimizu, T. Kobayashi, S. Odahara, G. Hougham, C. Cai, J. H. Glownia, R. J. von Gutfeld, R. John, and S.-C. A. Lien, "Development of one drop fill technology for AMLCDs,” SID Symp. Dig. Tech. Pap. 32, 1354-1357 (2001).

${ }^{3}$ Y. Yamamoto, "LCD processing and testing," in Handbook of Visual Display Technology, edited by J. Chen, W. Cranton, and M. Fihn (Springer, 2012), Vol. 3, Chap. 7.6.1, pp. 1649-1671.

${ }^{4}$ K.-C. Fan, J.-Y. Chen, C.-H. Wang, and W.-C. Pan, "Development of a drop-ondemand droplet generator for one-drop-fill technology," Sens. Actuators, A 147, 649-655 (2008).

${ }^{5}$ A. Hirai, I. Abe, M. Mitsumoto, and S. Ishida, "One drop filling for liquid crystal display panel produced for larger-sized mother glass," Hitachi Rev. 57, 144-148 (2008).

${ }^{6}$ W. K. Pratt, S. S. Sawkar, and K. O’Reilly, "Automatic blemish detection in liquid crystal flat panel displays,” Proc. SPIE 3306, 1-12 (1998).

${ }^{7}$ H. Lee, S. Song, J. Lee, A. Xiao, T. Min, and S. Su, "Analysis of liquid crystal drop mura in high resolution mobile thin film transistor liquid crystal displays," SID Symp. Dig. Tech. Pap. 45, 97-99 (2014).

${ }^{8}$ J. R. L. Cousins, S. K. Wilson, N. J. Mottram, D. Wilkes, L. Weegels, and K. Lin, "A model for the formation of mura during the one-drop-filling process," in Proceedings of the International Display Workshops, Volume 25, Nagoya, Japan, 12-14 December 2018 (Society for Information Display, 2018), pp. 125-127, Paper LCT8-4; available at: https://pureportal.strath.ac.uk/en/publications/a-model-forthe-formation-of-mura-during-the-one-drop-filling-pro.

${ }^{9} \mathrm{C}$. Zannoni, "Computer simulation and molecular design of model liquid crystals," in Chemistry at the Beginning of the Third Millennium, edited by L. Fabbrizzi and A. Poggi (Springer, 2000), pp. 329-342.

${ }^{10}$ C. M. Care and D. J. Cleaver, "Computer simulation of liquid crystals," Rep. Prog. Phys. 68, 2665-2700 (2005).

${ }^{11}$ R. W. Wilson, "Molecular simulation of liquid crystals: Progress towards a better understanding of bulk structure and the prediction of material properties," Chem. Soc. Rev. 36, 1881-1888 (2007).
${ }^{12}$ I. W. Stewart, The Static and Dynamic Continuum Theory of Liquid Crystals (Taylor and Francis, 2004).

${ }^{13}$ A. Sonnet, M. Virga, and G. Epifanio, Dissipative Ordered Fluids (Springer, 2012).

${ }^{14}$ P. G. De Gennes and J. Prost, The Physics of Liquid Crystals (Oxford University Press, 1993).

${ }^{15}$ J. L. Ericksen, “Anisotropic fluids," Arch. Ration. Mech. Anal. 4, 231-237 (1959).

${ }^{16}$ F. M. Leslie, "Some constitutive equations for anisotropic fluids," Q. J. Mech. Appl. Math. 19, 357-370 (1966).

${ }^{17}$ Y. Hori, Hydrodynamic Lubrication (Springer, 2006).

${ }^{18}$ L. Parry Jones, "Alignment properties of liquid crystals," in Handbook of Visual Display Technology, edited by J. Chen, W. Cranton, and M. Fihn (Springer, 2012), Vol. 3, Chap. 7.2.2, pp. 1387-1402.

${ }^{19}$ Physical Properties of Liquid Crystals: Nematics, edited by D. A. Dunmur, A. Fukuda, and G. R. Luckhurst (INSPEC, The Institution of Electrical Engineers, 2001).

${ }^{20} \mathrm{M}$. Miesowicz, "The three coefficients of viscosity of anisotropic liquids," Nature 158, 27 (1946).

${ }^{21}$ C.-T. Chen, "Inkjet printing of microcomponents: Theory, design, characteristics and applications," in Features of Liquid Crystal Display Materials and Processes, edited by N. Kamanina (IntechOpen, 2011), Chap. 3, pp. 43-60.

${ }^{22}$ J. Alamán, R. Alicante, J. I. Peña, and C. Sánchez-Somonlinos, "Inkjet printing of functional materials for optical and photonic applications," Materials 9,910 (2016).

${ }^{23}$ J. Quintans Carou, B. R. Duffy, N. J. Mottram, and S. K. Wilson, "Steady flow of a nematic liquid crystal in a slowly varying channel," Mol. Cryst. Liq. Cryst. 438, 237/1801-249/1813 (2005).

${ }^{24}$ J. Quintans Carou, B. R. Duffy, N. J. Mottram, and S. K. Wilson, "Shear-driven and pressure-driven flow of a nematic liquid crystal in a slowly varying channel," Phys. Fluids 18, 027105 (2006).

${ }^{25}$ J. Quintans Carou, N. J. Mottram, S. K. Wilson, and B. R. Duffy, "A mathematical model for blade coating of a nematic liquid crystal," Liq. Cryst. 34, 621-631 (2007).

${ }^{26}$ M. A. Lam, L. Cummings, T.-S. Lin, and L. Kondic, "Modeling flow of nematic liquid crystal down an incline," J. Eng. Math. 94, 97-113 (2015).

${ }^{27}$ M. Crespo, I. Griffiths, A. Majumdar, and A. Ramos, "Solution landscapes in nematic microfluidics," Physica D 351-352, 1-13 (2017).

${ }^{28}$ A. Rapini and M. Papoular, "Distorsion d'une lamelle nématique sous champ magnétique conditions d'ancrage aux parois," J. Phys. Colloq. 30, C4-54-C4-56 (1969). 